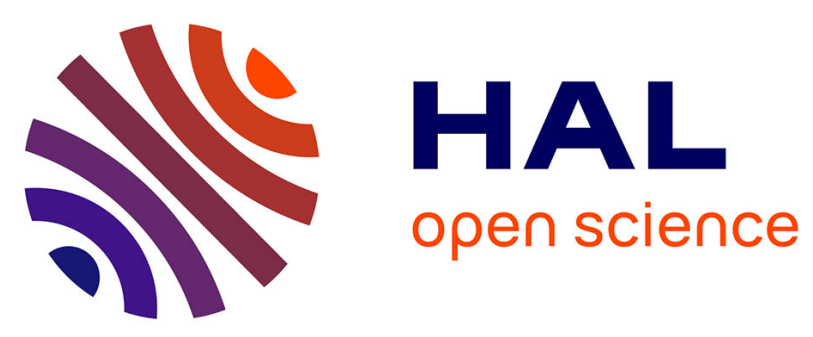

\title{
Phenolic, oxylipin and fatty acid profiles of the Chilean hazelnut (Gevuina avellana): Antioxidant activity and inhibition of pro-inflammatory and metabolic syndrome-associated enzymes
}

Liudis Leidy Pino Ramos, Felipe Jiménez-Aspee, Cristina Theoduloz, Alberto Burgos-Edwards, Raúl Domínguez-Perles, Camille Oger, Thierry Durand, Ángel Gil-Izquierdo, Luis Bustamante, Claudia Mardones, et al.

\section{- To cite this version:}

Liudis Leidy Pino Ramos, Felipe Jiménez-Aspee, Cristina Theoduloz, Alberto Burgos-Edwards, Raúl Domínguez-Perles, et al.. Phenolic, oxylipin and fatty acid profiles of the Chilean hazelnut (Gevuina avellana): Antioxidant activity and inhibition of pro-inflammatory and metabolic syndrome-associated enzymes. Food Chemistry, 2019, 298, pp.125026. 10.1016/j.foodchem.2019.125026 . hal-02617123

\author{
HAL Id: hal-02617123 \\ https://hal.science/hal-02617123
}

Submitted on 3 Jun 2021

HAL is a multi-disciplinary open access archive for the deposit and dissemination of scientific research documents, whether they are published or not. The documents may come from teaching and research institutions in France or abroad, or from public or private research centers.
L'archive ouverte pluridisciplinaire HAL, est destinée au dépôt et à la diffusion de documents scientifiques de niveau recherche, publiés ou non, émanant des établissements d'enseignement et de recherche français ou étrangers, des laboratoires publics ou privés. 


\title{
Phenolic, oxylipin and fatty acid profiles of the Chilean hazelnut (Gevuina avellana): Antioxidant activity and inhibition of pro-inflammatory and metabolic syndrome-associated enzymes
}

\author{
Liudis Leidy Pino Ramos ${ }^{\mathrm{a}}$, Felipe Jiménez-Aspee ${ }^{\mathrm{b}}$, Cristina Theoduloz ${ }^{\mathrm{c}}$, Alberto Burgos-Edwards ${ }^{\mathrm{a}}$, \\ Raúl Domínguez-Perles ${ }^{\mathrm{d}}$, Camille Oger ${ }^{\mathrm{e}}$, Thierry Durand ${ }^{\mathrm{e}}$, Ángel Gil-Izquierdo ${ }^{\mathrm{d}}$, \\ Luis Bustamante ${ }^{\mathrm{f}}$, Claudia Mardones ${ }^{\mathrm{f}}$, Katherine Márquez $^{\mathrm{g}}$, David Contreras ${ }^{\mathrm{g}}$, \\ Guillermo Schmeda-Hirschmann ${ }^{\text {g,* }}$ \\ ${ }^{a}$ Laboratorio de Química de Productos Naturales, Instituto de Química de Recursos Naturales, Universidad de Talca, Talca, Chile \\ ${ }^{\mathrm{b}}$ Departamento de Ciencias Básicas Biomédicas, Facultad de Ciencias de la Salud, Universidad de Talca, Talca, Chile \\ ${ }^{\mathrm{c}}$ Laboratorio de Cultivo Celular, Facultad de Ciencias de la Salud, Universidad de Talca, Talca, Chile \\ ${ }^{\mathrm{d}}$ Research Group on Quality, Safety and Bioactivity of Plant Foods, Department of Food Science and Technology, CEBAS (CSIC), University Campus of Espinardo, Edif. 25, \\ 30100 Murcia, Spain \\ ${ }^{\mathrm{e}}$ Institut des Biomolécules Max Mousseron, IBMM, UMR 5247, University of Montpellier, CNRS, ENSCM, Montpellier, France \\ ${ }^{\mathrm{f}}$ Departamento de Análisis Instrumental, Facultad de Farmacia, Universidad de Concepción, Chile \\ ${ }^{\mathrm{g}}$ Biotechnology Centre and Chemical Sciences Faculty, University of Concepcion, Concepcion, Chile
}

\section{A R T I C L E I N F O}

Chemical compounds studied in this article: $\alpha$-Linolenic acid (PubChem CID: 5280934) 5-Hydroxymethyl furfural (PubChem CID 237332)

7-Hexadecenoic acid (PubChem CID: 445639) Arachidonic acid (PubChem CID: 444899) Hydroxybenzoic acid (PubChem CID: 135)

Oleic acid (PubChem CID: 445639)

Keywords:

Chilean hazelnut

Gevuina avellana

Phenolics

Fatty acids

Phytoprostanes

Phytofurans

Polyunsaturated fatty acids

HPLC-MS/MS

\begin{abstract}
A B S T R A C T
Roasted cotyledons of the Chilean hazelnut (Gevuina avellana) are appreciated as snacks. The aim of our work was to assess the fatty acid, oxylipin and phenolic composition using gas chromatography (GC) coupled to mass spectrometry (MS), ultra- high performance liquid chromatography (UHPLC) coupled to MS and HPLC coupled to diode array detector (HPLC-DAD). Additionally, various antioxidant activities were assessed. The inhibition of $\alpha$-glucosidase, $\alpha$-amylase, lipase, cyclooxygenases-1 and -2 (COX-1/COX-2), and lipoxygenase was determined.

The main fatty acids were oleic and 7-hexadecenoic acids. Eight phytoprostanes and three phytofurans were identified and quantified. Hydroxybenzoic and hydroxycinnamic acids were the main phenolic compounds. Oils showed antioxidant activity determined by EPR, and inhibition of COX-1/COX-2. The statistical analysis showed that the roasting does not affect the composition of the samples. The occurrence of oxylipins in this species is reported for the first time. Chilean hazelnuts can be considered a source of health promoting compounds.
\end{abstract}

\section{Introduction}

Several nuts and seeds are relevant for human consumption including coconut (Cocos nucifera), peanut (Arachis hypogea) and the temperate species hazelnut (Corylus avellana), walnut (Juglans spp.) and almonds (Prunus amygdalis var. dulcis) (Gray, 2005). Nuts are

Abbreviations: AA, arachidonic acid; ALA, $\alpha$-linolenic acid; BHT, butylated hydroxytoluene; COX, cyclooxygenase; DPPH, 2,2-diphenyl-1-picrylhydrazyl radical; EPR, electron paramagnetic resonance; FAME, fatty acid methyl ester; FRAP, ferric reducing antioxidant power; GC-MS, gas chromatography-mass spectrometry; HPLC-MS/MS, high performance liquid chromatography coupled to mass spectrometry LOX, 15-lipoxygenase; MUFA, monounsaturated fatty acids; ORAC, oxygen radical absorbance capacity; PBN, N-t-butyl- $\alpha$-phenylnitrone; PEE, polyphenol-enriched extract; PhytoFs, phytofurans; PhytoPs, phytoprostanes; PUFAs, polyunsaturated fatty acids; SFA, saturated fatty acids; TEAC, Trolox equivalent antioxidant capacity; TP, total phenolic content

* Corresponding author.

E-mail address: schmeda@utalca.cl (G. Schmeda-Hirschmann). 
consumed either raw or roasted. Roasting adds additional organoleptic properties but can also modify the composition of health-promoting constituents (Taș and Gökmen, 2017; Chang, Alasalvar, Bolling, \& Shahidi, 2016). The Chilean hazelnut Gevuina avellana Mol. is the southernmost representative of the Proteaceae family, which includes the macadamia nut (Macadamia integrifolia and M. tetraphylla). The genus Macadamia is native to tropical Australia, and is cultivated in Hawaii, tropical Africa and South America (Uquiche, Jeréz, \& Ortíz, 2008; Gray, 2005). The common name Chilean hazelnut was given to $G$. avellana due to the morphological similarity with the European hazelnut Corylus avellana L. (Betulaceae).

In Chile, the gathering of $G$. avellana nuts can be traced back to preHispanic times. Remains of de-husked nuts are still frequently found in archeological sites. Mösbach (1992) described under the Mapuche name "gevuin" the tree Gevuina avellana, considered by the natives as the most relevant fruiting tree in their culture. The nuts are collected in March-April and are stored for the winter time. The fruits are de-husked and the seeds are roasted before consumption. Roasting is a traditional activity performed by small farmers or gatherers that collect and process the seeds for commercialization. They are obtained from wild stocks of the tree, either at the western Andean slopes or at the coastal range ("cordillera de la Costa"). Besides the consumption of roasted seeds, some by-products can be found in traditional markets. The cotyledons are grinded after mild or medium roasting to obtain a meal that is used for pastry products. If the cotyledons are intensively roasted, they can be grinded and used as a coffee substitute. Cotyledon oil is also used for cosmetic purposes (Flores García, Vergara, ForeroDoria, Guzmán, \& Pérez-Camino, 2018).

Most of the work performed so far on the Chilean hazelnut has been focused on the oil extracted from the seeds (Uquiche et al., 2008) and the thermal behavior of the oil (Flores García et al., 2018). Other studies include the extraction of antioxidants from $G$. avellana husks using different methods and antioxidant evaluation of the extracts (Moure, Franco, Sineiro, Domínguez, \& Núñez, 2003), but without any characterization of the phenolic constituents.

The deterioration of beverages and foods occur through oxidation reactions that are generally promoted by free radicals. Antioxidants present in these foods can stop or trap these radicals, thus inhibiting the oxidative damage of foods. Currently, the antioxidant activity of beverages and foods is mainly measured by spectrophotometric methods. The electron paramagnetic resonance (EPR) spectroscopy with spin trapping is a technique that uses a direct method to evaluate the reduction of a stable adduct (Polovka, 2006; Papadimitriou et al., 2006). The adduct is produced by photochemical excitation, generating a more stable radical product (spin adduct). The EPR spectroscopy is used in biology and medicine for the direct detection of radical species formed during oxidative stress and via enzymatic reactions. The compound $\mathrm{N}-\mathrm{t}$ butyl- $\alpha$-phenylnitrone (PBN) has been successfully employed as a spin trap for the entrapment of lipid free radicals in food lipids (Thomsen, Kristensen, \& Skibsted, 2000) and vegetable oils (Papadimitriou et al., 2006; Velasco, Andersen, \& Skibsted, 2004).

The polyunsaturated fatty acids (PUFAs) are relevant molecules in eukaryotic organisms and contribute critically to the cell membrane structure and integrity. They are also precursors of a series of compounds prone to cell signaling functions in mammals and higher plants (Durand et al., 2011). In the latter, bioactive lipids include phytoprostanes (PhytoPs) and phytofurans (PhytoFs) derived from the oxidation of $\alpha$-linolenic acid (ALA) (Durand et al., 2011). These oxylipins (PhytoPs and PhytoFs) are associated with cell response to oxidative damage and might play a role as mediators of an array of pathophysiological conditions (Durand et al., 2011). Thus, in general, PhytoPs and PhytoFs content increases as a result of the exposition of stressing conditions and display relevant bioactivities in plants, including signaling and detoxification actions (Loeffler et al., 2005). The potential biological functions of ALA and oxylipins in humans after dietary ingestion have been proposed by Durand et al. (2011). However, this has not been completely demonstrated so far.

While the fatty acid composition of some G. avellana samples has been described, there is no information on the occurrence of oxylipins and polyphenols, neither the potential health benefits from the different constituents of the nut. In addition, G. avellana cotyledons are roasted before consumption, and this thermal process may affect the content and composition of phytochemicals. In the light of the current knowledge, our hypothesis is that G. avellana nuts are a source of fatty acids, oxylipins and polyphenols with potential health promoting activities, which can be affected by the roasting process. The aim of the present work was to gain a further insight in the fatty acid and phenolic composition of Chilean hazelnuts, as well as the occurrence of PhytoPs and PhytoFs in this food matrix. In addition, the antioxidant activity, as well as the inhibition towards pro-inflammatory and metabolic syndromeassociated enzymes was studied. Raw and roasted material was included to determine if the roasting process affects the content, composition and activity of the samples.

\section{Materials and methods}

\subsection{Chemicals and reagents}

2,2'-Azino-bis(3-ethylbenzothiazoline-6-sulfonic acid) diammonium salt (ABTS), 6-hydroxy-2,5,7,8-tetramethylchroman-2-carboxilic acid (Trolox), potassium persulfate, sodium carbonate, Folin-Ciocalteu reagent, acetic acid, $\mathrm{FeCl}_{3} \times 6 \mathrm{H}_{2} \mathrm{O}$, acetonitrile (ACN), methanol $(\mathrm{MeOH})$, chloroform, linolenic acid, $\mathrm{BF}_{3}$, anhydrous $\mathrm{Na}_{2} \mathrm{SO}_{4}$, potassium sodium tartrate and formic acid were purchased from Merck (Darmstadt, Germany). Sodium chloride, potassium phosphate monobasic, sodium hydroxide were purchased from Scharlau (Barcelona, Spain). Acarbose, Amberlite XAD-7HP resin, bis-tris, butylated hydroxytoluene (BHT), 2,2-diphenyl-1-picrylhydrazyl radical (DPPH), 3,5- dinitrosalicylic acid, 4-nitrophenyl- $\alpha$-D-glucopyranoside, 4-nitrophenyl palmitate, starch, Triton X-100, 2,4,6-tri(2-pyridyl)1,3,5-triazine (TPTZ), potassium chloride, lipase from porcine pancreas (L3126; EC 3.1.1.3), $\alpha$-amylase from porcine pancreas (A3176; EC 3.2.1.1), a-glucosidase from Saccharomyces cerevisiae (G5003; EC 3.2.1.20), L-glutamine, and lipoxygenase (LOX Type I-B from Glycine max, L73953) were obtained from Sigma-Aldrich (St. Louis, MO, USA). Orlistat was from Laboratorio Chile (Santiago, Chile). The $\alpha$-phenyl- $N$-tert-butylnitrone (PBN) was from Axxora (Enzo Life Sciences, Farmingdale, NY, USA). $N$-hexane was acquired from Panreac (Barcelona, Spain). HPLC-MS grade solvents were from J.T. Baker (Phillipsburg, NJ, USA).

Standard fatty acids methyl esters mix for quantification Supelco 37 Component fatty acid methyl esters (FAME) mix, Sigma-Aldrich (St. Louis, MO, USA). The standards of PhytoPs and PhytoFs were supplied by the Institut des Biomolécules Max Mousseron (IBMM, Montpellier, France). They were synthesized as previously described (El Fangour et al., 2004; Guy, Flanagan, Durand, Oger, \& Galano, 2015; Cuyamendous et al., 2017). Ultrapure water was obtained from a Barnstead EasyPure water system (Thermo Scientific, Marietta, OH, USA).

\subsection{Plant material}

The samples analyzed included unprocessed (raw) and roasted cotyledons from Chilean hazelnut (Gevuina avellana), ensuring that the raw and roasted samples were from the same collection. In addition, a coffee substitute and meal prepared from the roasted cotyledons were included in this study. The oil obtained from raw and roasted cotyledons was also studied. Samples were collected in central-southern Chile, between $35^{\circ} 35^{\prime} \mathrm{S}, 71^{\circ} 12^{\prime} \mathrm{W}$ and $41^{\circ} 51^{\prime} \mathrm{S}, 71^{\circ} 54^{\prime} \mathrm{W}$, as follows: Región del Maule, raw (2 samples) and roasted cotyledons (1 sample); Región de Nuble, raw (2 samples) and roasted cotyledons (4 samples) and meal (1 sample); Región del Bío-Bío, raw (1 sample) and roasted cotyledons ( 5 samples by "callana" roasting process, and 4 samples by 
"tostador" roasting process), coffee substitute (1 sample) and meal (1 sample); Región de Los Lagos: raw cotyledons (1 sample). Local producers from Contulmo (Región del Bío-Bío) declared 2 different roasting procedures, namely: "callana" and "tostador". For the "callana" process, a container usually made from metal is used to roast the grains. The original "callana" roasting was made in a clay container, but afterwards it was substituted by a metal vessel. The "tostador" roasting is usually performed on a metal plate (iron, zinc or copper). The samples collected in the other regions did not specify the type of roasting. The sample from Región de Los Lagos was raw material collected in the wild.

\subsection{Extraction and sample preparation}

The raw and roasted cotyledons, as well as the meal and coffee substitute samples were purchased from rural cooperatives. The samples were transported to the laboratory and stored at room temperature $\left(15-20^{\circ} \mathrm{C}\right)$ until processing within 4 weeks. The $G$. avellana material was separately processed to obtain the oil (for fatty acids and PhytoPs/ PhytoFs analyses) and the polyphenol-enriched extracts (PEE). The resulting PEEs and oils were kept at $4{ }^{\circ} \mathrm{C}$.

\subsubsection{Oil extraction from Gevuina avellana nuts}

Dried samples (200 g) were homogenized in a blender (Thomas TH$501 \mathrm{~V}$, Thomas Elektrogeräte, Shanghai, China) and then extracted three times with hexane $(3 \times 800 \mathrm{~mL})$ for $15 \mathrm{~min}$ in an ultrasonic bath $(35 \mathrm{kHz})$ at room temperature (Elma Transonic 700, Elma GmbH \& Co. KG, Singen, Germany). The combined solution was filtered, concentrated under reduced pressure at $37^{\circ} \mathrm{C}$ using a rotary evaporator (Büchi, Flawil, Switzerland), dehydrated with anhydrous sodium sulfate and taken to dryness to afford the lipid fraction.

\subsubsection{Polyphenols extraction from Gevuina avellana nuts}

The defatted material was further extracted with $\mathrm{MeOH}$ $(3 \times 500 \mathrm{~mL})$ for $15 \mathrm{~min}$ in an ultrasonic bath. The $\mathrm{MeOH}$ solution was filtered, taken to dryness under reduced pressure, re-suspended in water and extracted with chloroform to remove remaining lipids. After removal of the residual chloroform in the aqueous phase, the watersoluble solution was mixed with Amberlite XAD-7 in an Amberlite:extract 5:1 ratio for $40 \mathrm{~min}$ under constant stirring. After filtering, the Amberlite was rinsed with distilled water and compounds were eluted with $\mathrm{MeOH}$. The eluate was dried under reduced pressure below $40{ }^{\circ} \mathrm{C}$ to afford the PEE used for the study.

\subsection{Fatty acids analysis from Gevuina avellana oils}

The fatty acid content and composition of the different oils was assessed following the AOAC 969.33 protocol for fatty acids, after derivatization as fatty acid methyl esters (FAME) (Heldrich, 2000). Linolenic acid $(0.91 \mathrm{~g} / \mathrm{L})$ was used as the internal standard. Briefly, the oils were saponified with $\mathrm{NaOH}(0.5 \mathrm{M})$ and treated with $\mathrm{BF}_{3}$ to prepare the methyl esters for analysis. Oil samples $(500 \mu \mathrm{L})$ were pooled to build a quality control (QC). The QC was used to optimize the chromatographic separation. The QC was injected every three samples to evaluate the reproducibility of the analytical method.

The GC-MS analysis was performed using an ionic liquid IL-111 column $(30 \mathrm{~m} \times 0.25 \mathrm{~mm} \times 0.2 \mu \mathrm{m}$, polarity similar to that of Carbowax). Injection temperature: $250^{\circ} \mathrm{C}$, split mode $25: 1$, flow: $1.5 \mathrm{~mL} / \mathrm{min}$. Oven temperature program: starting at $40^{\circ} \mathrm{C}$ for $4 \mathrm{~min}$, $5{ }^{\circ} \mathrm{C} / \mathrm{min}$ ramp to $210^{\circ} \mathrm{C}$. Analysis time: $38 \mathrm{~min}+4 \mathrm{~min}$ post-run at $240^{\circ} \mathrm{C}$. Transference line: $270{ }^{\circ} \mathrm{C}$. Ionization source: $70 \mathrm{eV}$ and $230^{\circ} \mathrm{C}$. Quadrupole temperature: $150{ }^{\circ} \mathrm{C}$.

Readings were performed in the scan mode from 40 to $400 \mathrm{~m} / \mathrm{z}$, with 8.4 cycles/s. Fatty acids were identified by comparing the retention times with a FAME mix and the mass spectra.

Extracted ions at $m / z 74$ and 87 were used for the saturated and monounsaturated fatty acids. The ions at $m / z 79$ and 81 were used for the di- and tri-unsaturated fatty acids (Thurnhofer $\&$ Vetter, 2005). The areas corrected by the internal standard of the samples and the standard mix (Supelco pn: CRM47885, lot: XA18647V) were compared applying a response factor method, according to the AOAC 996.06, using the following formula:

$F i=\frac{A i * C_{I S}}{A_{I S} * R i}$

where: $\mathrm{Fi}=$ concentration of individual fatty acid in the sample; $\mathrm{Ai}=$ extracted ion chromatogram (EIC) peak area of the individual fatty acid in the sample as FAME; $\mathrm{C}_{\mathrm{IS}}=$ concentration $(\mathrm{g} / \mathrm{L})$ of internal standard in the sample; $\mathrm{A}_{\mathrm{IS}}=$ EIC peak area of internal standard in the sample; and $\mathrm{Ri}=$ response factor for each fatty acid. The Ri was calculated according to the following formula:

$R i=\frac{A s i * C s_{I S}}{A s_{I S} * C s i}$

where: Asi = EIC peak area of the individual FAME in the standard mix; $\mathrm{Cs}_{\mathrm{IS}}=$ analytical concentration $(\mathrm{g} / \mathrm{L})$ of internal standard in the standard mix; $\mathrm{As}_{\mathrm{IS}}=\mathrm{EIC}$ peak area of internal standard in the standard mix, and $\mathrm{Csi}=$ analytical concentration $(\mathrm{g} / \mathrm{L})$ of the individual FAME in the standard mix. The concentration of the different fatty acids was expressed as $\mathrm{g} / \mathrm{L}$ oil and as percentage of the total content.

\subsection{Electron paramagnetic resonance (EPR) assays of the oils from $G$. avellana}

The reduction of the radical products from the starting $N$ - $t$-butyl- $\alpha$ phenylnitrone (PBN) spin trap (PBN-ox) was determined by means of electron paramagnetic resonance (EPR) assays. The antioxidant activity of seven selected $G$. avellana oils, including roasted and raw samples, was evaluated. The spectra were measured in a Bruker model EMXmicro-spectrometer (Bruker GmbH, Mannheim, Germany). The reactor contained $1.1 \mathrm{~mL}$ of $G$. avellana oil and $0.6 \mathrm{~mL}$ of PBN $100 \mathrm{mM}$ in cyclohexane. The chemical oxidation process of the PBN was accelerated by UV radiation for $180 \mathrm{~s}$. The instrumentation and conditions were as follows. Measurements were carried out in a flat quartz cell, EPR microwave power: $2.000 \mathrm{~mW}$, modulation frequency $100 \mathrm{kHz}$, sweep time: 30 s, number of scans: 10; receiver gain: $30 \mathrm{~dB}$; sweep width: 100 $\mathrm{G}$ and 3475 as the center field for experiments with PBN as the spin trapping was used. For the quantification of the signal, the double integral under the peak and the height of the peak of greater intensity were used. The results were expressed as intensity of the signal and area obtained.

\subsection{Phytoprostanes (PhytoPs) and phytofurans (PhytoFs) profile and concentration in $G$. avellana nuts}

The extraction of PhytoPs and PhytoFs was developed according to Carrasco-Del Amor et al. (2017). Briefly, samples (5 g) were homogenized in a blender and extracted with a $1 \mathrm{~g} / \mathrm{L}$ solution of butylated hydroxytoluene (BHT) in $\mathrm{MeOH}$. The solution was stirred for $10 \mathrm{~min}$ and centrifuged at $13,000 \mathrm{rpm}$ for $15 \mathrm{~min}$ at $4{ }^{\circ} \mathrm{C}$ to recover the supernatant. This solution was partitioned with chloroform:bis-tris $(8: 2, \mathrm{v} / \mathrm{v}$, $\mathrm{pH}=7.0$ ) and stirred for further $15 \mathrm{~min}$ at room temperature. The solution was centrifuged and the organic phase was recovered and divided into two fractions for subsequent analysis. One fraction was submitted to a basic hydrolysis with $1 \mathrm{M} \mathrm{KOH}$, and then to an acid hydrolysis with $0.04 \mathrm{M}$ formic acid. The other fraction was dried and reconstituted with hexane:bis-tris $(2: 1, \mathrm{v} / \mathrm{v}, \mathrm{pH} 7.0)$. Both fractions were submitted to a solid phase extraction using a Strata X-AW cartridge (Phenomenex, Torrance, CA, USA) according to the procedure described by Collado-Gonzalez et al. (2015). The PhytoPs and PhytoFs were recovered with $1 \mathrm{~mL}$ of $\mathrm{MeOH}$, dried and reconstituted in $200 \mu \mathrm{L}$ of the mobile phase described below. 
Twenty $\mu \mathrm{L}$ of reconstituted PhytoPs and PhytoFs were injected and analyzed in a UHPLC coupled with a 6460 triple quadrupole-MS/MS (Agilent Technologies, Waldbronn, Germany) by applying the analytical settings described by Collado-Gonzalez et al. (2015). The analytical column was a BEH C18 $(2.1 \times 50 \mathrm{~mm}, 1.7 \mu \mathrm{m})$ (Waters, Milford, MA, USA), maintained at $6{ }^{\circ} \mathrm{C}$. The mobile phases consisted of water/ acetic acid (99.99:0.01, v/v, solvent A) and methanol/acetic acid (99.99:0.01, v/v, solvent B). The flow rate was $0.2 \mathrm{~mL} / \mathrm{min}$ and the linear gradient was as follows: $t=0 \mathrm{~min}, 60 \% \mathrm{~B} ; \mathrm{t}=2 \mathrm{~min}, 62 \% \mathrm{~B}$; $\mathrm{t}=4 \mathrm{~min}, 62.5 \% \mathrm{~B} ; \mathrm{t}=8 \mathrm{~min}, 65 \% \mathrm{~B} ; \mathrm{t}=8.01 \mathrm{~min}, 60 \% \mathrm{~B}$. A post-run of $1.5 \mathrm{~min}$ was allowed for the column equilibration. The MS parameters for fragmentation of PhytoPs and PhytoFs were set according to Collado-Gonzalez et al. (2015). The quantification was achieved using external calibration curves with authentic standards. The regression coefficient for the calibration curves of the PhytoPs and PhytoFs were 0.999, 0.999, 0.998, 0.995, 0.999, 0.999, 0.999, 0.999, 0.992, 0.999, and 0.999 for $9-\mathrm{F}_{1 \mathrm{t}}-\mathrm{PhytoP}$, 9-epi-9- $\mathrm{F}_{1 \mathrm{t}}-\mathrm{PhytoP}$, ent- $16-\mathrm{F}_{1 \mathrm{t}}-\mathrm{PhytoP}$, ent-

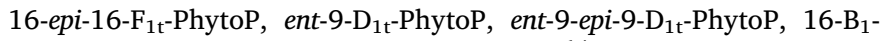
PhytoP, 9- $\mathrm{L}_{1}-\mathrm{PhytoP}$, ent-16-(RS)-9-epi-ST- $\Delta^{14}-10-\mathrm{PhytoF}$, ent-9-(RS)12-epi-ST- $\Delta^{10}-13$-PhytoF, and ent-16-(RS)-13-epi-ST- $\Delta^{14}-9-$ PhytoF, respectively. The linearity ranges for all analytes was found between 0.59 and $344.44 \mathrm{ng} / \mathrm{mL}$. Samples were appropriately diluted to fit within this range of concentrations. Intra-day variations of the instrument sensitivity were determined by analyzing three replicates of the calibration curves at the start, intermediate and at the end of the analysis. Analyses were carried out in triplicate and the PhytoPs and PhytoFs content was expressed as $\mathrm{ng} / 100 \mathrm{~g}$ cotyledon $\pm \mathrm{SD}$.

\subsection{Total phenolic (TP) content in the polyphenol enriched extract from $G$. avellana nuts}

The total phenolic (TP) content of the PEE was quantified spectrophotometrically by the Folin-Ciocalteau method as previously reported (Burgos-Edwards, Jiménez-Aspee, Thomas-Valdés, SchmedaHirschmann, \& Theoduloz, 2017). Briefly, stock solutions of the PEEs were dissolved in $\mathrm{MeOH}(1-5 \mathrm{mg} / \mathrm{mL})$ and an aliquot was mixed with the Folin-Ciocalteu reagent. Then, the volume was adjusted to $20 \mathrm{~mL}$ with distilled water and $1 \mathrm{~mL}$ of $\mathrm{Na}_{2} \mathrm{CO}_{3}(20 \% \mathrm{w} / \mathrm{v})$ was added and completed to $25 \mathrm{~mL}$ with distilled water. After $60 \mathrm{~min}$, absorbance was measured at $725 \mathrm{~nm}$ in a Genesys 10UV (Thermo Spectronic, Rochester, NY, USA). Each sample was assayed in triplicate and results were expressed as mean values $\pm \mathrm{SD}$. Values are presented as $\mathrm{g}$ of gallic acid equivalent (GAE)/100 $\mathrm{g}$ of PEE.

\subsection{HPLC-DAD and HPLC-MS/MS analysis of polyphenols from $G$. avellana nuts}

The PEE extracts were analyzed by HPLC coupled to a diode array detector (DAD) to establish conditions for HPLC-MS/MS measurements. Chromatograms were recorded using a Shimadzu (Shimadzu Corporation, Kyoto, Japan) chromatograph consisting of an LC-20AT pump, a SPD-M20A diode array detector, a CTO-20AC oven and LabSolution software. An Inertsil ODS-3 RP-18 column (GL Sciences Inc., Tokyo, Japan; $250 \mathrm{~mm} \times 4.6 \mathrm{~mm}$ and $5 \mu \mathrm{m}$ of particle size) was used. Temperature: $22^{\circ} \mathrm{C}$; flow rate: $0.8 \mathrm{~mL} / \mathrm{min}$; detection: UV, $280 \mathrm{~nm}$. The solvent system used was methanol (solvent A) and $\mathrm{H}_{2} \mathrm{O}$ :formic acid (solvent B, 99.9:0.1 v/v). The chromatographic conditions started from $5 \%$ A: $95 \%$ B from 0 to 2 min, $20 \%$ A: $80 \%$ B at $8 \mathrm{~min} ; 52 \%$ A: $48 \%$ B at $40 \mathrm{~min} ; 75 \%$ A: $25 \%$ B at $45 \mathrm{~min}: 100 \%$ A from 50 to $55 \mathrm{~min}$ and return to $5 \% \mathrm{~A}$ and $95 \% \mathrm{~B}$ at $60 \mathrm{~min}$. The chromatograms were monitored at 254, 280 and $330 \mathrm{~nm}$ and the UV/visible spectra were recorded from 200 to $600 \mathrm{~nm}$ for the characterization of chromophores from the eluted compounds.

HPLC-MS/MS analysis were performed using an HPLC HP 1100 equipment (Agilent Technologies Inc., Santa Clara, CA) connected through a split to a LC/MS trap system (Esquire 4000, Bruker Daltoniks,
Bremen, Germany). The data were acquired in the full scan mode (range of $m / z 150-2000$ ) operated in the negative mode. Nitrogen was used as nebulizer gas at $27.5 \mathrm{psi}, 350^{\circ} \mathrm{C}$ and at a flow rate of $8 \mathrm{~L} / \mathrm{min}$. The conditions for the analysis were as follows: electrospray needle, $4000 \mathrm{~V}$; compensating end plate, $-500 \mathrm{~V}$; separator cone $1,56.0 \mathrm{~V}$; separator cone 2, 6.0 V; output compensation capillary, $84.6 \mathrm{~V}$; and output capillary, $140.6 \mathrm{~V}$. The collision induced dissociation spectra were obtained with a fragmentation amplitude of $1.00 \mathrm{~V}$ (MS/MS) using helium as the colliding gas. The compounds were identified by comparison of the observed fragmentation pattern and UV profiles with standards and compounds reported in literature.

\subsection{Nuclear magnetic resonance (NMR) analysis of the polyphenol- enriched extract (PEEs) from G. avellana nuts}

The PEE of the samples were analyzed by NMR $\left({ }^{1} \mathrm{H}, 400 \mathrm{MHz}\right)$ to confirm the occurrence of compounds tentatively assigned by HPLCMS/MS, and to identify main compounds not detected by mass spectrometry under our working conditions. The NMR spectra were recorded on a Bruker Avance 400 spectrometer (Bruker BioSpin, Rheinstetten, Germany) at $400 \mathrm{MHz}$ for ${ }^{1} \mathrm{H}$ and $100 \mathrm{MHz}$ for ${ }^{13} \mathrm{C}$ in $\mathrm{MeOH}-d_{4}$. Chemical shifts are given in ppm with residual methanol as the internal standard.

\subsection{Antioxidant activity of the polyphenol-enriched extracts (PEE) from G. avellana nuts}

The antioxidant activity of the PEEs was determined by the following assays: discoloration of the 2,2-diphenyl-1-picrylhydrazyl (DPPH) and the 2,2'-azino-bis(3-ethylbenzothiazoline-6-sulfonic acid diammonium salt (ABTS) radicals (Trolox equivalent antioxidant capacity, TEAC), the ferric reducing antioxidant power (FRAP) and oxygen radical antioxidant capacity (ORAC) assays. All the methodologies were carried out as previously described (Jiménez-Aspee et al., 2018). Stock solutions of the PEEs were dissolved in $\mathrm{MeOH}$ (1-5 mg/ $\mathrm{mL}$, for the DPPH, FRAP and TEAC assays) or phosphate buffer $(10-50 \mu \mathrm{g} / \mathrm{mL}$, for the ORAC assay). Samples were assayed in triplicate in all determinations, and the results are presented as mean values \pm SD. Quercetin was used as the positive control in all the assays.

For the DPPH assay, an aliquot of the sample was mixed with the DPPH radical $(20 \mathrm{mg} / \mathrm{L})$ and after $5 \mathrm{~min}$ the absorbance was determined in a universal microplate reader at $515 \mathrm{~nm}$ (Biotek Instruments Inc., ELx 800, Winooski, VT, USA). Results are expressed as the amount of sample that is able to scavenge the radical by $50 \%\left(\mathrm{SC}_{50}\right.$, $\mu \mathrm{g} / \mathrm{mL}$ ).

For the FRAP assay, an aliquot of the sample was mixed with the FRAP solution ( $300 \mathrm{mM}$ acetate buffer, $\mathrm{pH} 3.6,20 \mathrm{mM} \mathrm{FeCl}_{3} \mathrm{x}_{6} \mathrm{H}_{2} \mathrm{O}$ and $10 \mathrm{mM}$ TPTZ in a 10:1:1. ratio). After $30 \mathrm{~min}$, absorbance was measured in a Genesys 10UV (Thermo Spectronic, Rochester, NY, USA) at $593 \mathrm{~nm}$. Results are expressed as mmol Trolox equivalents (TE)/g PEE.

In the TEAC assay, the ABTS radical was prepared by mixing $140 \mathrm{mM} \mathrm{K}_{2} \mathrm{~S}_{2} \mathrm{O}_{8}$ with $7.5 \mathrm{mM}$ ABTS. After $16 \mathrm{~h}$, the radical was diluted with $\mathrm{MeOH}$ to reach an absorbance of $0.700 \pm 0.005$ at $734 \mathrm{~nm}$. An aliquot of the samples was mixed with $2.85 \mathrm{~mL}$ of the radical solution and absorbances were measured after $6 \mathrm{~min}$. The results are expressed as $\mu \mathrm{M} \mathrm{TE} / \mathrm{g}$ PEE.

In the ORAC assay, the effect of the samples in the decline of the fluorescence $\left(\lambda_{\text {em }}=515 \mathrm{~nm}\right.$; $\left.\lambda_{\text {ex }}=493 \mathrm{~nm}\right)$ of fluorescein $(0.11 \mu \mathrm{M})$ in the presence of the 2,2'-Azobis(2-methylpropionamidine) (AAPH) radical $(153 \mathrm{mM})$ was measured every min during $90 \mathrm{~min}$ in a microplate reader (Synergy HTX, Biotek Instruments Inc., Winooski, VT, USA). The assay was carried out at $37^{\circ} \mathrm{C}$ in the dark. The results were expressed as $\mu \mathrm{mol} \mathrm{TE} / \mathrm{g}$ PEE. 
Table 1

Fatty acid composition of G. avellana oils (g/L).

\begin{tabular}{|c|c|c|c|c|c|c|c|}
\hline Sample & $\mathrm{C} 16: 0$ & $\mathrm{C} 16: 1[1]$ & $\mathrm{C} 18: 0$ & C18:1n9 & C18:1 [2] & $\mathrm{C} 18: 2 \mathrm{n} 9,12$ & C20:0 \\
\hline \multicolumn{8}{|l|}{ Región del Maule } \\
\hline Vilches, 2016A & $17.5 \pm 2.0^{\mathrm{a}}$ & $193.4 \pm 13.5^{\mathrm{a}}$ & $3.9 \pm 0.6^{\mathrm{ab}}$ & $327.6 \pm 25.3^{\mathrm{a}}$ & $46.3 \pm 3.8^{\mathrm{a}}$ & $60.6 \pm 5.5^{\mathrm{a}}$ & $15.2 \pm 1.9^{\mathrm{a}}$ \\
\hline Vilches, 2017A & $11.4 \pm 1.3^{\mathrm{b}}$ & $188.1 \pm 13.1^{\mathrm{a}}$ & $2.8 \pm 0.4^{\mathrm{a}}$ & $271.5 \pm 20.9^{\mathrm{a}}$ & $48.2 \pm 3.9^{\mathrm{a}}$ & $42.0 \pm 3.8^{\mathrm{b}}$ & $10.5 \pm 1.3^{\mathrm{b}}$ \\
\hline Vilches, 2016B & $14.6 \pm 1.7^{\mathrm{ab}}$ & $166.4 \pm 11.6^{\mathrm{a}}$ & $4.6 \pm 0.7^{b}$ & $281.0 \pm 21.7^{\mathrm{a}}$ & $49.3 \pm 4.0^{\mathrm{a}}$ & $49.4 \pm 4.5^{\mathrm{ab}}$ & $16.2 \pm 2.1^{\mathrm{a}}$ \\
\hline \multicolumn{8}{|l|}{ Región de Ñuble } \\
\hline Los Lleuques, 2017A, April & $11.4 \pm 1.3^{\mathrm{a}}$ & $178.4 \pm 12.4^{\mathrm{abc}}$ & $2.0 \pm 0.3^{\mathrm{a}}$ & $264.3 \pm 20.4^{\mathrm{a}}$ & $45.0 \pm 3.6^{\mathrm{a}}$ & $45.3 \pm 4.1^{\mathrm{a}}$ & $8.7 \pm 1.1^{\mathrm{a}}$ \\
\hline Los Lleuques, 2017A, May & $11.3 \pm 1.3^{\mathrm{a}}$ & $169.1 \pm 11.8^{\mathrm{b}}$ & $3.1 \pm 0.5^{\mathrm{a}}$ & $257.1 \pm 19.8^{\mathrm{a}}$ & $45.4 \pm 3.7^{\mathrm{a}}$ & $44.9 \pm 4.1^{\mathrm{a}}$ & $11.4 \pm 1.5^{\mathrm{a}}$ \\
\hline Los Lleuques, 2016B & $15.7 \pm 1.8^{\mathrm{abc}}$ & $189.1 \pm 13.2^{\mathrm{abc}}$ & $3.0 \pm 0.5^{\mathrm{a}}$ & $295.2 \pm 22.8^{\mathrm{abc}}$ & $48.6 \pm 3.9^{\mathrm{ab}}$ & $58.7 \pm 5.4^{\mathrm{b}}$ & $11.5 \pm 1.5^{\mathrm{a}}$ \\
\hline Los Lleuques, 2017B, April & $13.3 \pm 1.5^{\mathrm{abc}}$ & $199.4 \pm 13.9^{\mathrm{abc}}$ & $3.4 \pm 0.5^{\mathrm{ac}}$ & $297.2 \pm 22.9^{\mathrm{abc}}$ & $52.4 \pm 4.2^{\mathrm{ab}}$ & $51.6 \pm 4.7^{\mathrm{ab}}$ & $12.2 \pm 1.6^{\mathrm{a}}$ \\
\hline Los Lleuques, 2017B, May & $16.7 \pm 1.9^{c}$ & $211.3 \pm 14.7^{c}$ & $4.7 \pm 0.8^{\mathrm{bc}}$ & $335.3 \pm 28.9^{c}$ & $59.6 \pm 4.8^{\mathrm{bc}}$ & $51.9 \pm 4.7^{\mathrm{ab}}$ & $16.7 \pm 2.1^{\mathrm{a}}$ \\
\hline Los Lleuques, Meal, 2016B & $12.8 \pm 1.5^{\mathrm{abc}}$ & $174.8 \pm 12.2^{\mathrm{b}}$ & $3.1 \pm 0.5^{\mathrm{a}}$ & $276.0 \pm 21.3^{\mathrm{abc}}$ & $47.5 \pm 3.8^{\mathrm{a}}$ & $45.9 \pm 4.2^{\mathrm{ab}}$ & $11.4 \pm 1.5^{\mathrm{a}}$ \\
\hline Yungay, Meal, 2016B & $15.1 \pm 1.8^{\mathrm{abc}}$ & $175.5 \pm 12.2^{\mathrm{abc}}$ & $3.2 \pm 0.5^{\mathrm{a}}$ & $284.6 \pm 21.9^{\mathrm{abc}}$ & $48.1 \pm 3.9^{\mathrm{a}}$ & $56.5 \pm 5.2^{\mathrm{ab}}$ & $11.8 \pm 1.5^{\mathrm{a}}$ \\
\hline \multicolumn{8}{|l|}{ Región del Bío-Bío } \\
\hline Contulmo, 2017A & $17.3 \pm 2.0^{\mathrm{ac}}$ & $200.1 \pm 13.9^{\mathrm{abcd}}$ & $5.5 \pm 0.9^{b}$ & $332.7 \pm 25.6^{\mathrm{a}}$ & $53.5 \pm 4.3^{\mathrm{ad}}$ & $59.6 \pm 5.4^{\mathrm{a}-\mathrm{j}}$ & $17.5 \pm 2.2^{\mathrm{a}}$ \\
\hline Contulmo, Meal, 2016B & $17.7 \pm 2.0^{\mathrm{a}}$ & $195.5 \pm 13.6^{\mathrm{abcd}}$ & $4.2 \pm 0.7^{\mathrm{a}-\mathrm{d}}$ & $312.3 \pm 24.1^{\mathrm{ab}}$ & $49.8 \pm 4.0^{\mathrm{a}-\mathrm{e}}$ & $70.4 \pm 6.4^{\mathrm{a}}$ & $15.4 \pm 2.0^{\mathrm{abc}}$ \\
\hline Contulmo, Coffee, 2016B & $11.7 \pm 1.4^{\text {bceim }}$ & $173.4 \pm 12.1^{\mathrm{a}-\mathrm{i}}$ & $3.0 \pm 0.5^{\mathrm{acd}}$ & $270.9 \pm 20.9^{\mathrm{ab}}$ & $44.0 \pm 3.6^{\mathrm{abce}}$ & $51.4 \pm 4.7^{\text {cgdef }}$ & $11.7 \pm 1.5^{\mathrm{bcd}}$ \\
\hline Contulmo, 2016B1, April & $17.2 \pm 2.0^{\mathrm{ap}}$ & $170.0 \pm 11.8^{\text {befg }}$ & $4.6 \pm 0.7^{\mathrm{bd}}$ & $301.5 \pm 23.2^{\mathrm{ab}}$ & $47.3 \pm 3.8^{\mathrm{a}-\mathrm{e}}$ & $54.7 \pm 5.0^{\text {bdefgh }}$ & $16.0 \pm 2.0^{\mathrm{abc}}$ \\
\hline Contulmo, 2016B1, May & $12.6 \pm 1.5^{\mathrm{cfjnp}}$ & $196.1 \pm 13.6^{\text {aceh }}$ & $3.6 \pm 0.6^{\mathrm{acd}}$ & $296.6 \pm 22.9^{\mathrm{ab}}$ & $49.3 \pm 4.0^{\mathrm{a}-\mathrm{e}}$ & $57.1 \pm 5.2^{\mathrm{a}-\mathrm{j}}$ & $13.1 \pm 1.7^{\mathrm{a}-\mathrm{d}}$ \\
\hline Contulmo, 2017B1, April & $13.2 \pm 1.5^{\text {abcgko }}$ & $199.0 \pm 13.8^{\text {aceh }}$ & $3.1 \pm 0.5^{\mathrm{acd}}$ & $298.8 \pm 23.0^{\mathrm{ab}}$ & $51.9 \pm 4.2^{\mathrm{a}-\mathrm{e}}$ & $57.7 \pm 5.3^{\mathrm{a}-\mathrm{j}}$ & $12.1 \pm 1.5^{\mathrm{bcd}}$ \\
\hline Contulmo, 2017B1, May & $15.5 \pm 1.8^{\mathrm{abcd}}$ & $211.1 \pm 14.7^{\mathrm{c}}$ & $3.8 \pm 0.6^{\mathrm{a}-\mathrm{d}}$ & $307.0 \pm 23.7^{\mathrm{ab}}$ & $55.0 \pm 0.5^{\mathrm{d}}$ & $64.7 \pm 5.9^{\mathrm{agi}}$ & $15.1 \pm 1.9^{\mathrm{abc}}$ \\
\hline Contulmo, 2016B2, April & $11.0 \pm 1.3^{\operatorname{defgh}}$ & $179.8 \pm 12.5^{\mathrm{a}-\mathrm{i}}$ & $3.0 \pm 0.5^{\mathrm{acd}}$ & $275.7 \pm 21.3^{\mathrm{ab}}$ & $44.0 \pm 3.6^{\text {abce }}$ & $48.4 \pm 4.4^{\mathrm{dj}}$ & $11.7 \pm 1.5^{\mathrm{bcd}}$ \\
\hline Contulmo, 2016B2, May & $11.8 \pm 1.4^{\mathrm{dijkl}}$ & $155.8 \pm 10.8^{\mathrm{f}}$ & $3.2 \pm 0.5^{\mathrm{acd}}$ & $252.9 \pm 19.5^{\mathrm{b}}$ & $42.7 \pm 3.5^{\text {abce }}$ & $52.4 \pm 4.8^{\mathrm{eij}}$ & $11.6 \pm 1.5^{\mathrm{bcd}}$ \\
\hline Contulmo, 2017B2, April & $10.4 \pm 1.2^{\text {hlmno }}$ & $164.7 \pm 11.5^{\mathrm{dfhi}}$ & $2.4 \pm 0.4^{\mathrm{c}}$ & $248.4 \pm 19.1^{\mathrm{b}}$ & $41.6 \pm 3.4^{\mathrm{b}}$ & $45.9 \pm 4.2^{\mathrm{fj}}$ & $9.9 \pm 1.3^{\mathrm{d}}$ \\
\hline Contulmo, 2017B2, May & $13.8 \pm 1.6^{\text {abcdgko }}$ & $202.2 \pm 14.1^{\mathrm{acgi}}$ & $3.4 \pm 0.5^{\mathrm{acd}}$ & $293.4 \pm 22.6^{\mathrm{ab}}$ & $52.5 \pm 4.3^{\mathrm{de}}$ & $65.0 \pm 5.9^{\mathrm{ahi}}$ & $14.3 \pm 1.8^{\mathrm{a}-\mathrm{d}}$ \\
\hline Quality control $^{1}$ & $15.2 \pm 1.8$ & $190.2 \pm 13.2$ & $4.0 \pm 0.6$ & $304.3 \pm 23.5$ & $50.6 \pm 4.1$ & $55.7 \pm 5.1$ & $14.1 \pm 1.8$ \\
\hline Sample & $\mathrm{C} 20: 1 \mathrm{n} 11$ & C20:1 [3] & $\mathrm{C} 21: 0$ & $\mathrm{C} 22: \ln 13$ & $\mathrm{C} 22: 1[4]$ & $\mathrm{C} 24: 0$ & Total \\
\hline \multicolumn{8}{|l|}{ Región del Maule } \\
\hline Vilches, 2016A & $22.1 \pm 3.1^{\mathrm{a}}$ & $48.3 \pm 4.5^{\mathrm{a}}$ & $16.8 \pm 3.3^{\mathrm{a}}$ & $22.8 \pm 6.2^{\mathrm{a}}$ & $124.4 \pm 17.4^{\mathrm{a}}$ & $2.7 \pm 1.3^{\mathrm{a}}$ & $901.4 \pm 35.4^{\mathrm{a}}$ \\
\hline Vilches, 2017A & $14.6 \pm 2.1^{b}$ & $43.2 \pm 4.0^{\mathrm{a}}$ & $10.8 \pm 2.1^{\mathrm{a}}$ & $16.7 \pm 3.5^{\mathrm{a}}$ & $99.8 \pm 13.9^{\mathrm{a}}$ & $1.9 \pm 0.9^{\mathrm{a}}$ & $757.5 \pm 29.6^{\mathrm{b}}$ \\
\hline Vilches, 2016B & $17.0 \pm 2.4^{\mathrm{a}}$ & $47.2 \pm 4.4^{\mathrm{a}}$ & $17.3 \pm 3.4^{\mathrm{a}}$ & $17.3 \pm 4.7^{\mathrm{a}}$ & $112.5 \pm 15.7^{\mathrm{a}}$ & $2.7 \pm 1.2^{\mathrm{a}}$ & $795.7 \pm 30.9^{\mathrm{b}}$ \\
\hline \multicolumn{8}{|l|}{ Región de Nuble } \\
\hline Los Lleuques, 2017A, April & $14.9 \pm 2.1^{\mathrm{a}}$ & $39.9 \pm 3.7^{\mathrm{a}}$ & $8.6 \pm 1.7^{\mathrm{a}}$ & $10.5 \pm 2.9^{\mathrm{a}}$ & $94.3 \pm 13.2^{\mathrm{a}}$ & $1.7 \pm 0.8^{\mathrm{ab}}$ & $724.9 \pm 28.4^{\mathrm{ad}}$ \\
\hline Los Lleuques, 2017A, May & $15.9 \pm 2.2^{\mathrm{a}}$ & $43.5 \pm 4.0^{\mathrm{a}}$ & $11.5 \pm 2.3^{\mathrm{a}}$ & $15.8 \pm 4.3^{\mathrm{ab}}$ & $104.6 \pm 14.6^{\mathrm{a}}$ & $2.5 \pm 1.2^{\mathrm{ab}}$ & $734.0 \pm 28.7^{\mathrm{ad}}$ \\
\hline Los Lleuques, 2016B & $15.4 \pm 2.2^{\mathrm{a}}$ & $48.2 \pm 4.0^{\mathrm{ab}}$ & $11.0 \pm 2.2^{\mathrm{a}}$ & $12.4 \pm 3.4^{\mathrm{a}}$ & $101.5 \pm 14.2^{\mathrm{a}}$ & $1.8 \pm 0.9^{\mathrm{ab}}$ & $807.1 \pm 31.3^{\mathrm{cd}}$ \\
\hline Los Lleuques, 2017B, April & $17.2 \pm 2.4^{\mathrm{ab}}$ & $48.8 \pm 4.5^{\mathrm{ab}}$ & $11.7 \pm 2.3^{\mathrm{a}}$ & $15.9 \pm 4.4^{\mathrm{ab}}$ & $114.0 \pm 15.9^{\mathrm{ab}}$ & $2.8 \pm 1.3^{\mathrm{ab}}$ & $839.8 \pm 32.7^{c}$ \\
\hline Los Lleuques, 2017B, May & $22.9 \pm 3.2^{\mathrm{b}}$ & $57.7 \pm 5.3^{\mathrm{b}}$ & $22.0 \pm 4.4^{\mathrm{b}}$ & $25.8 \pm 7.1^{\mathrm{b}}$ & $153.1 \pm 21.4^{\mathrm{b}}$ & $4.3 \pm 2.0^{\mathrm{a}}$ & $981.9 \pm 38.8^{\mathrm{b}}$ \\
\hline Los Lleuques, Meal, 2016B & $15.5 \pm 2.2^{\mathrm{a}}$ & $43.1 \pm 4.0^{\mathrm{a}}$ & $12.1 \pm 2.4^{\mathrm{a}}$ & $12.0 \pm 3.3^{\mathrm{a}}$ & $102.5 \pm 14.3^{\mathrm{a}}$ & $1.7 \pm 0.8^{\mathrm{ab}}$ & $758.7 \pm 29.7^{\mathrm{cd}}$ \\
\hline Yungay, Meal, 2016B & $14.5 \pm 2.0^{\mathrm{a}}$ & $43.6 \pm 4.0^{\mathrm{a}}$ & $11.3 \pm 2.2^{\mathrm{a}}$ & $12.7 \pm 3.5^{\mathrm{a}}$ & $101.7 \pm 14.2^{\mathrm{a}}$ & $0.9 \pm 0.4^{\mathrm{b}}$ & $779.3 \pm 30.3^{\mathrm{cd}}$ \\
\hline \multicolumn{8}{|l|}{ Región del Bío-Bío } \\
\hline Contulmo, 2017A & $21.8 \pm 3.0^{\mathrm{ad}}$ & $49.7 \pm 4.6^{\mathrm{a}-\mathrm{c}}$ & $19.0 \pm 3.8^{\mathrm{a}}$ & $20.1 \pm 5.5^{\mathrm{a}}$ & $122.7 \pm 17.1^{\mathrm{ab}}$ & $3.3 \pm 1.5^{\mathrm{a}}$ & $922.7 \pm 35.8^{\mathrm{a}}$ \\
\hline Contulmo, Meal, 2016B & $20.3 \pm 2.8^{\mathrm{a}-\mathrm{e}}$ & $50.2 \pm 4.6^{\mathrm{a}-\mathrm{c}}$ & $18.9 \pm 3.7^{\mathrm{a}}$ & $23.2 \pm 6.3^{\mathrm{a}}$ & $138.6 \pm 19.4^{\mathrm{a}}$ & $3.2 \pm 1.5^{\mathrm{a}}$ & $919.6 \pm 35.9^{\mathrm{a}}$ \\
\hline Contulmo, Coffee, 2016B & $15.5 \pm 2.2^{\mathrm{a}-\mathrm{e}}$ & $41.8 \pm 3.9^{\mathrm{a}-\mathrm{c}}$ & $10.0 \pm 2.0^{\mathrm{b}}$ & $10.5 \pm 2.9^{\mathrm{a}}$ & $95.0 \pm 13.3^{\mathrm{ab}}$ & $0.8 \pm 0.4^{\mathrm{a}}$ & $739.9 \pm 28.8^{\mathrm{bd}}$ \\
\hline Contulmo, 2016B1, April & $20.6 \pm 2.9^{\mathrm{bd}}$ & $45.6 \pm 4.2^{\mathrm{a}-\mathrm{c}}$ & $16.2 \pm 3.2^{\mathrm{ab}}$ & $21.1 \pm 5.8^{\mathrm{a}}$ & $109.7 \pm 15.3^{\mathrm{ab}}$ & $2.9 \pm 1.4^{\mathrm{a}}$ & $827.3 \pm 32.2^{\text {be }}$ \\
\hline Contulmo, 2016B1, May & $18.8 \pm 2.6^{\mathrm{a}-\mathrm{e}}$ & $48.3 \pm 4.5^{\mathrm{a}-\mathrm{c}}$ & $12.7 \pm 2.5^{\mathrm{ab}}$ & $17.5 \pm 4.8^{\mathrm{a}}$ & $127.5 \pm 17.8^{\mathrm{ab}}$ & $2.5 \pm 1.2^{\mathrm{a}}$ & $855.7 \pm 33.6^{\text {aef }}$ \\
\hline Contulmo, 2017B1, April & $15.3 \pm 2.1^{\mathrm{a}-\mathrm{e}}$ & $47.4 \pm 4.4^{\mathrm{a}-\mathrm{c}}$ & $11.9 \pm 2.4^{\mathrm{ab}}$ & $11.4 \pm 3.1^{\mathrm{a}}$ & $102.7 \pm 14.3^{\mathrm{ab}}$ & $0.9 \pm 0.4^{\mathrm{a}}$ & $825.4 \pm 31.9^{\mathrm{bfg}}$ \\
\hline Contulmo, 2017B1, May & $20.0 \pm 2.8^{\mathrm{a}-\mathrm{e}}$ & $52.8 \pm 4.9^{\mathrm{ac}}$ & $13.8 \pm 2.7^{\mathrm{ab}}$ & $18.6 \pm 5.1^{\mathrm{a}}$ & $121.0 \pm 16.9^{\mathrm{ab}}$ & $2.6 \pm 1.2^{\mathrm{a}}$ & $900.7 \pm 34.5^{\text {aeg }}$ \\
\hline Contulmo, 2016B2, April & $15.8 \pm 2.2^{\mathrm{a}-\mathrm{e}}$ & $42.5 \pm 3.9^{\mathrm{a}-\mathrm{c}}$ & $13.7 \pm 2.7^{\mathrm{ab}}$ & $15.0 \pm 4.1^{\mathrm{a}}$ & $109.5 \pm 15.3^{\mathrm{ab}}$ & $1.9 \pm 0.9^{\mathrm{a}}$ & $772.2 \pm 30.4^{\mathrm{bfh}}$ \\
\hline Contulmo, 2016B2, May & $14.1 \pm 2.0^{\text {be }}$ & $39.1 \pm 3.6^{\mathrm{b}}$ & $11.0 \pm 2.2^{\mathrm{ab}}$ & $11.3 \pm 3.1^{\mathrm{a}}$ & $82.8 \pm 11.6^{\mathrm{b}}$ & $1.7 \pm 0.8^{\mathrm{a}}$ & $690.2 \pm 26.5^{\mathrm{cdh}}$ \\
\hline Contulmo, 2017B2, April & $13.3 \pm 1.9^{\text {ce }}$ & $38.4 \pm 3.6^{\mathrm{b}}$ & $9.6 \pm 1.9^{b}$ & $10.8 \pm 3.0^{\mathrm{a}}$ & $86.9 \pm 12.1^{\mathrm{b}}$ & $1.5 \pm 0.7^{\mathrm{a}}$ & $683.6 \pm 26.6^{\mathrm{cdh}}$ \\
\hline Contulmo, 2017B2, May & $17.6 \pm 2.5^{\mathrm{a}-\mathrm{e}}$ & $52.2 \pm 4.8^{\mathrm{c}}$ & $14.3 \pm 2.8^{\mathrm{ab}}$ & $14.7 \pm 4.0^{\mathrm{a}}$ & $128.8 \pm 18.0^{\mathrm{ab}}$ & $2.7 \pm 1.0^{\mathrm{a}}$ & $874.4 \pm 33.9^{\text {aeg }}$ \\
\hline Quality control $^{1}$ & $19.0 \pm 2.7$ & $48.7 \pm 4.5$ & $15.7 \pm 3.1$ & $18.8 \pm 5.1$ & $122.9 \pm 17.2$ & $2.6 \pm 1.2^{\mathrm{a}}$ & $861.8 \pm 33.7$ \\
\hline
\end{tabular}

A: raw; B: roasted; B1 "callana" roasting process; B2: "tostador" roasting process [1]: as C16:1n9 (Z) equivalents, [2]: as C18:1n9 (Z) equivalents; [3]: as C20:1n11 (Z) equivalents; [4]: as C22:1n13 (Z) equivalents; ${ }^{1} \mathrm{n}=10$, corresponds to CV (\%) obtained for QC assuming homocedasticity of the results variability of all measured analytes. *coffee substitute prepared by toasting the seeds until color resembles coffee beans. The G. avellana meal is obtained by grinding the roasted cotyledons. Results are presented as mean values $\pm \mathrm{SD}(\mathrm{g} / \mathrm{L} ; \mathrm{n}=10)$. Different lower case letters (a-i) on each column show significant differences according to Tukey's test $(p<0.05)$.

\subsection{Enzyme inhibition studies}

\subsubsection{Inhibition of metabolic syndrome-associated enzymes by the PEEs} from G. avellana

The PEEs from the G. avellana were assessed as inhibitors of $\alpha$ amylase, $\alpha$-glucosidase and pancreatic lipase, by means of colorimetric assays as described by Burgos-Edwards et al. (2017).

In the lipase assay, the substrate was $p$-nitrophenyl palmitate $(0.08 \%, \mathrm{w} / \mathrm{v})$ and the reaction mixture was incubated at $37^{\circ} \mathrm{C}$ during $2 \mathrm{~h}$. Absorbance was measured in a spectrophotometer at $400 \mathrm{~nm}$. In the $\alpha$-glucosidase assay, the substrate was $p$-nitrophenyl- $\alpha$-D-glucopyranoside $(0.15 \%, \mathrm{w} / \mathrm{v})$, and incubated at $37^{\circ} \mathrm{C}$ for $30 \mathrm{~min}$. Absorbances were measured in a microplate reader at $405 \mathrm{~nm}$. In the $\alpha$-amylase assay, the substrate was starch $(1 \%, \mathrm{w} / \mathrm{v})$. The reaction mixture was incubated at $37^{\circ} \mathrm{C}$ for $40 \mathrm{~min}$. Absorbances were read in a spectrophotometer at $550 \mathrm{~nm}$.

Acarbose and orlistat were used as the reference compounds. The PEEs were assessed at maximum concentrations of 100 and $50 \mu \mathrm{g}$ PEE/ $\mathrm{mL}$. Results are expressed as percentage of inhibition or as the amount of sample that is able to inhibit the enzyme by $50 \%\left(\mathrm{IC}_{50}, \mu \mathrm{g} / \mathrm{mL}\right)$. 


\subsubsection{Inhibition of pro-inflammatory enzymes by G. avellana oils}

The inhibitory activity of seven selected oils from G. avellana was determined against 15-lipoxygenase (LOX) and cyclooxygenase-1 and -2 (COX-1, COX-2). Oils were dissolved in dimethyl sulfoxide (DMSO) and diluted to a final concentration of $100 \mu \mathrm{g} / \mathrm{mL}$ in reaction buffer. The $100 \%$ activity controls were carried out using DMSO and buffer. Indomethacin and naproxen were used as the reference compounds.

The LOX inhibition assay was carried out according to Wangensteen, Samuelsen, and Malterud (2004). The substrate was linoleic acid $(134 \mu \mathrm{M})$ and the oxidation of the substrate by the enzyme was followed during $90 \mathrm{~s}$ at $234 \mathrm{~nm}$ in a spectrophotometer. The assay was carried out in triplicate and results are expressed as percentage of inhibition \pm SD.

The inhibition of COX-1 and COX-2 was carried out using a commercial kit (Cayman Chemicals, $\mathrm{N}^{\circ}$ 560131, Ann Arbor, MI, USA), following the supplier instructions. The prostaglandin $\mathrm{F}_{2 \alpha}$ production was measured at $415 \mathrm{~nm}$ in a microplate reader. Samples were evaluated in triplicate and results are expressed as percentage of inhibition \pm SD.

\subsection{Statistical analyses}

The statistical analyses were carried out using the SPSS 14.0 software (IBM, Armonk, NY, USA). Statistically significant differences within each region of collection were determined by one-way analysis of variance (ANOVA) followed by Tukey's multiple comparison test $(p<0.05)$. The correlation between the quantified components (TP and fatty acids) and the anti-inflammatory as well as the antioxidant activity was analyzed by the Pearson's correlation coefficient with $95 \%$ confidence.

To determine if the roasting process induces changes in the chemical content and composition, a principal component analysis (PCA) was carried out using the Pirouette v.4.5 software package (Infometrix, Bothell, WA, USA). The descriptors for the raw and roasted samples were the content of fatty acids, oxylipins, antioxidant activity assays (DPPH, FRAP, TEAC, ORAC and EPR), inhibition of enzymes ( $\alpha$-amylase, $\alpha$-glucosidase, lipase, COX-1, COX-2 and LOX), the yield of PEE and the total phenolic content. Thirty eight independent variables were used and the data were normalized. The model was corroborated by cross-validation.

\section{Results and discussion}

Chilean hazelnuts (23 samples) purchased in local markets and collected in Región del Maule, Región de Ñuble, Región del Bío-Bío and Región de Los Lagos were analyzed. It is important to point out that the raw and roasted samples were from the same collection, to ensure that the differences observed were due to the roasting process.

\subsection{Fatty acids analysis from the G. avellana oils}

The oil content of the G. avellana cotyledons ranged from 42 to $51 \%$ $\mathrm{w} / \mathrm{w}$ (data not shown). The individual fatty acid content and composition of the oils, determined as FAME, was assessed by GC-MS. The fatty acids occurring in the oils are listed in Table S1 with the retention time $\left(R_{t}, \min \right)$ and the ions used for quantification/identification. The content of fatty acids $(\mathrm{g} / \mathrm{L})$ is presented in Table 1 , including the corresponding statistical analysis. In addition, the total content of saturated fatty acids (SFA), monounsaturated fatty acids (MUFA) and polyunsaturated fatty acids (PUFA) is summarized in Table S2. Thirteen fatty acids were identified and quantified. A representative chromatogram is presented in Fig. S1. Two compounds co-eluted with a very small difference in the $\mathrm{R}_{\mathrm{t}}(27.36$ and $27.37 \mathrm{~min})$. According to the elution order and mass spectra, they were identified as linoleic and arachidic acid, respectively (Table S1). The most abundant fatty acids in the G. avellana oil samples were MUFAs, in particular C18:1n9 (oleic acid) and C16:1 (7-hexadecenoic acid), with contents ranging from
248.4-335.3 and from 155.8-211.1 g/L, respectively. Flores García et al. (2018) reported that these fatty acids were the main components of the oil from G. avellana samples collected in the Nahuelbuta coastal mountains (Región del Bío-Bío), representing about 23.6 and $36.8 \%$ of the total content, for C16:1 and C18:1n9, respectively. A study on the $G$. avellana oil purchased at the Región de la Araucanía, reported a high fatty acids content accounting for $93.14 \%$ of the total weight. The authors quantified individual fatty acids as methyl esters and reported with that the content of C18:1n9 and C16:1n9 was 36.3 and $29.1 \%$, respectively (Uquiche et al., 2008). On the other hand, Birch, Yap, and Silcock (2010) compared the fatty acid composition of G. avellana and Macadamia spp., and showed that in both species the main fatty acids were $\mathrm{C} 16: 1 \mathrm{n} 11$ and $\mathrm{C}: 18: 1 \mathrm{n} 9$. The main difference between both genus was the high content of $\mathrm{C} 22: \ln 17$ in G. avellana. In the same way, our results noticed C22:1 as the third most abundant fatty acid in the oils evaluated (Table 1).

A principal component analysis (PCA) was carried out in order to evaluate the effect of the roasting process on the fatty acid composition. A total of 38 independent variables were used considering two different approaches. The first approach was to assess if there were differences between raw and roasted samples, while the second approach was to evaluate if there were differences among the geographical regions of collection. The results of both analyses showed that there were no differences within the samples, neither by the geographical origin (Fig. S2) nor by the roasting process (Fig. S3). In agreement with our results, a recent study about the effect of the roasting process on the oil obtained from three poppy seed varieties showed non-significant changes in the content and composition of the fatty acids (Ghafoor, Özcan, ALJuhaimi, Babiker, \& Fadimu, 2019).

\subsection{Fatty acid antioxidant activity of the oils from $G$. avellana}

The antioxidant activity of the selected oils was determined by EPR. In order to identify the formed adduct, the spectrum of the PBN-ox adduct was performed using the Xenon software (Bruker). Although the simulated spectra showed two hyperfine division constants at $\alpha \mathrm{N}=14.7 \mathrm{G}$ and $\alpha \mathrm{H}=2.5 \mathrm{G}$, the experimental spectra shows only the constant at $\alpha \mathrm{N}=13.8 \mathrm{G}$ (Fig. S4). The absence of the signal at $\alpha \mathrm{H}=2.5$ could be explained by the reduced mobility of the radicals due to the long chain of oxidized lipids, or the high viscosity of the medium (Davies, 1987). PBN-ox is a stable adduct and was measured up to $15 \mathrm{~h}$. Therefore, the signal of this adduct was used for the EPR analysis (Fig. S4). The PBN-ox adduct has been reported in the literature as a reaction product between ${ }^{\circ} \mathrm{OH}$ with PBN (Papadimitriou et al., 2006).

All samples were subjected to UV radiation for $180 \mathrm{~s}$ to induce the radical formation. The PBN-ox control showed significant differences with all the samples, according to Tukey's multiple comparison test $(p<0.05)$ (Table 2). However, only some samples exhibited significant differences among them. Under our experimental conditions, the highest antioxidant effect, considering the area of the PBN-ox, was found in the raw and roasted oil samples from Región del Maule, while the less antioxidant samples where from Región del Bío-Bío (Table 2). This means that the samples from Región del Maule are more resistant to the formation of free radicals, whereas the samples from Región del Bío-Bío seem to be more susceptible to free radical generation. A significant correlation between the content of polyphenols and the EPR induction time was reported for virgin olive oil (Papadimitriou et al., 2006). Our results also evidenced a significant correlation between the TP content and the area under the curve of the PBN-ox signal $(\mathrm{r}=0.640, p<0.01)$ and the intensity of the signal $(\mathrm{r}=0.517$, $p<0.05$ ). Moreover, Flores García et al. (2018) showed that despite $G$. avellana oil presents a lower content of polyphenols compared with olive oil, the antioxidant activity, by means of the DPPH discoloration assay, is similar after thermal stress. 
Table 2

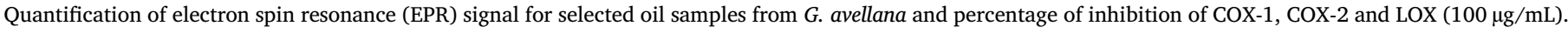

\begin{tabular}{|c|c|c|c|c|c|}
\hline \multirow[t]{2}{*}{ Sample } & \multicolumn{2}{|l|}{ EPR } & \multirow[t]{2}{*}{ COX-1 (\%) } & \multirow[t]{2}{*}{ COX-2 (\%) } & \multirow[t]{2}{*}{ LOX (\%) } \\
\hline & Intensity & Area & & & \\
\hline \multicolumn{6}{|l|}{ Región del Maule } \\
\hline Vilches A & $0.07 \pm 0.01^{\mathrm{ab}}$ & $1.28 \pm 0.06^{\mathrm{a}}$ & $50.1 \pm 3.3^{\mathrm{a}}$ & $15.7 \pm 1.2^{\mathrm{a}}$ & $10.3 \pm 1.7^{\mathrm{a}}$ \\
\hline Vilches B & $0.05 \pm 0.01^{\mathrm{ab}}$ & $1.58 \pm 0.29^{\mathrm{ab}}$ & inactive & $33.8 \pm 3.2^{\mathrm{b}}$ & $21.2 \pm 3.4^{\mathrm{b}}$ \\
\hline \multicolumn{6}{|l|}{ Región de Ñuble } \\
\hline Los Lleuques A & $0.07 \pm 0.01^{\mathrm{ab}}$ & $2.12 \pm 0.06^{\mathrm{b}}$ & $79.9 \pm 0.3^{b}$ & $17.4 \pm 1.2^{\mathrm{a}}$ & $9.1 \pm 2.8^{\mathrm{a}}$ \\
\hline Los Lleuques B & $0.08 \pm 0.01^{\mathrm{bd}}$ & $2.36 \pm 0.35^{\mathrm{b}}$ & inactive & $20.6 \pm 2.2^{\mathrm{a}}$ & $20.0 \pm 3.8^{\mathrm{b}}$ \\
\hline \multicolumn{6}{|c|}{ Región del Bío-Bío } \\
\hline Contulmo A & $0.12 \pm 0.01^{\mathrm{c}}$ & $3.47 \pm 0.14^{\mathrm{c}}$ & $73.1 \pm 3.3^{\mathrm{b}}$ & inactive & $26.0 \pm 1.0^{\mathrm{b}}$ \\
\hline Contulmo B1 & $0.08 \pm 0.00^{\mathrm{ad}}$ & $2.15 \pm 0.03^{b}$ & inactive & inactive & $25.2 \pm 0.6^{\mathrm{b}}$ \\
\hline Contulmo B2 & $0.11 \pm 0.02^{\mathrm{cd}}$ & $3.29 \pm 0.60^{c}$ & $71.8 \pm 4.8^{\mathrm{b}}$ & inactive & $22.0 \pm 1.0^{\mathrm{b}}$ \\
\hline Indometacin* & - & - & $0.08 \pm 0.01$ & $0.01 \pm 0.00$ & - \\
\hline Naproxen* & - & - & - & - & $14.0 \pm 0.7$ \\
\hline PBN-ox & $1.98 \pm 0.02$ & $113.40 \pm 0.66$ & - & - & - \\
\hline
\end{tabular}

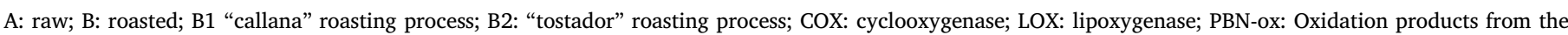

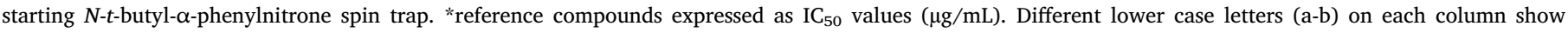
significant differences according to Tukey's test $(p<0.05)$.

\subsection{Phytoprostane (PhytoPs) and phytofuran (PhytoFs) content in G. avellana nuts}

The exposition of plants to biotic and abiotic stress increases the production of PUFA derivatives upon oxidative reactions. Under this conditions, free radicals are directed against ALA, generating prostanoid-like structures named PhytoPs. The addition of molecular oxygen after initial cyclization leads to the generation of furanic structures called PhytoFs. The nomenclature described by Taber, Fessel, and Roberts (2004) was used in this work, which was approved by IUPAC for unequivocally naming the multitude of structures.

The presence and content of PhytoPs and PhytoFs in G. avellana kernels was studied. The content of these compounds is shown in Table 3 and the structures are presented in Fig. S5. Eight different PhytoPs were detected and quantified in raw and roasted samples of $G$. avellana. The main PhytoPs in the studied samples were $9-\mathrm{F}_{1 \mathrm{t}}$-PhytoP and 9-epi-9- $\mathrm{F}_{1 \mathrm{t}}$-PhytoP, with contents ranging from 0-393.8 and 0-1515.42 ng/100 g for raw and roasted cotyledons, respectively. Interestingly, the content of 9-epi-9- $\mathrm{D}_{1 \mathrm{t}}$-PhytoP and 9- $\mathrm{D}_{1 \mathrm{t}}$-PhytoP was significantly higher in the G. avellana meal purchased in Región del BíoBío, while in most of the other samples these PhytoPs were not detected. The coffee substitute showed the highest content of ent-16-epi$16-\mathrm{F}_{1 \mathrm{t}}-\mathrm{PhytoP}+$ ent-16- $\mathrm{F}_{1 \mathrm{t}}-\mathrm{PhytoP}(p<0.05)$. In three out of the four collection regions, the hydrolysis of the raw cotyledons led to a significant increase in the PhytoP content. On the other hand, no significant effect on the PhytoP content was observed after the roasting process. PhytoPs have been previously identified and quantified in vegetable oils. Collado-Gonzalez et al. (2015) reported the content of PhytoPs and PhytoFs in plant oils, focusing their study on the detection and quantification of free PhytoPs. The PhytoPs content of refined sunflower oil was higher than in virgin olive oil, suggesting that the processing has a relevant effect on the synthesis of oxylipins from the PUFA precursors in the samples (Collado-Gonzalez et al., 2015). Carrasco-Del Amor et al. (2017) reported the changes in the PhytoP content and composition induced by the frying process of different nut kernels, including the related macadamia nuts. The total PhytoPs content in raw pecan, macadamia, and walnut kernels were 7830.3, 6465.5 and $5541.5 \mathrm{ng} / \mathrm{kg}$, respectively. The content of PhytoPs found in macadamia nuts was increased about five times after frying. A similar trend was also observed in pecan nuts. The main PhytoP found in these samples was 9-F-PhytoPs (Carrasco-Del Amor et al., 2017).

In the present study the occurrence of PhytoFs in raw and roasted samples of G. avellana was also monitored. The PhytoFs content and composition is shown in Table 3. Most PhytoFs occurring in our samples are in the esterified form and are released after hydrolysis. The roasting process rises the content of ent-16-(RS)-13-epi-ST- $\Delta^{14}-9$-PhytoF and total PhytoFs formed as a consequence of the fatty acids oxidation. Regarding the two different roasting processes, "callana" increased the PhytoFs content mainly providing higher amounts of ent-16-(RS)-12epi-ST- $\Delta^{10}-13-P h y t o F$ and ent-16-(RS)-13-epi-ST- $\Delta^{14}-9-P h y t o F$. However, in the roasted cotyledons from the sample "Contulmo 2017B1, April", PhytoFs were not detected. The hydrolysis allowed the detection of PhytoFs in this sample. In the coffee substitute, the content of ent-16(RS)-13-epi-ST- $\Delta^{14}$-9-PhytoF increased after the hydrolysis, while the ent-16-(RS)-12-epi-ST- $\Delta^{10}$-13-PhytoF disappeared after hydrolysis. In the meal sample from Yungay 2016B, only the ent-16-(RS)-9-epi-ST- $\Delta^{14}$ 10 -PhytoF was detected after hydrolysis. On the other hand, all the PhytoFs were identified in the meal from "Contulmo 2016B", and the content of ent-16-(RS)-9-epi-ST- $\Delta^{14}-10-$ PhytoF significantly increased after the hydrolysis, while the other PhytoFs were reduced to trace levels. Interestingly, the "tostador" samples from year 2016 and 2017 were characterized by the presence of the ent-16-(RS)-9-epi-ST- $\Delta^{14}-10$ PhytoF, while the "callana" samples showed different levels of the three PhytoFs. Some differences were observed depending on the collection place, however these differences were not significant according to the PCA analysis (Fig. S2).

Phytofurans are much less studied than PhytoPs. Cuyamandous et al. (2015) described the content and composition of these compounds in pine nuts, walnuts, flaxseeds and chia seeds. In another study, LeónPerez et al. (2019) reported the content of PhytoFs in the beans from 31 cocoa clones. The content of PhytoFs ranged from 1.18 to $13.13 \mathrm{ng} / \mathrm{g}$, showing high variability according to the environmental conditions (León-Perez et al., 2019).

\subsection{Total phenolic content (TP) in the polyphenol-enriched extracts (PEEs) from $G$. avellana nuts}

The yield of the PEE was found between 0.01-1.04 g/100 g dried cotyledons (Table 4). The TP content in the Chilean hazelnut cotyledons PEE was low (Table 4). The content ranged from 1.9 to $4.6 \mathrm{~g} \mathrm{GAE} / 100 \mathrm{~g}$ $\mathrm{PEE}$ in raw samples. The roasted samples showed values between 1.6-8.6 g GAE/100 g PEE. The coffee substitute showed a TP content of 8.7 g GAE/100 g PEE, while the three meal samples showed TP values in the range of $2.1-8.3 \mathrm{~g} \mathrm{GAE} / 100 \mathrm{~g}$ PEE. Paradoxically, the roasting process can either increase or decrease the TP content. The increase may be due to the release of bound phenolics from the food matrix, while the decrease may be due to the degradation of phenolic compounds by high temperatures (Taş \& Gökmen, 2017). The roasting 
Table 3

Phytoprostane (PhytoPs) and phytofuran (PhytoFs) content (ng/100 g) in G. avellana cotyledons.

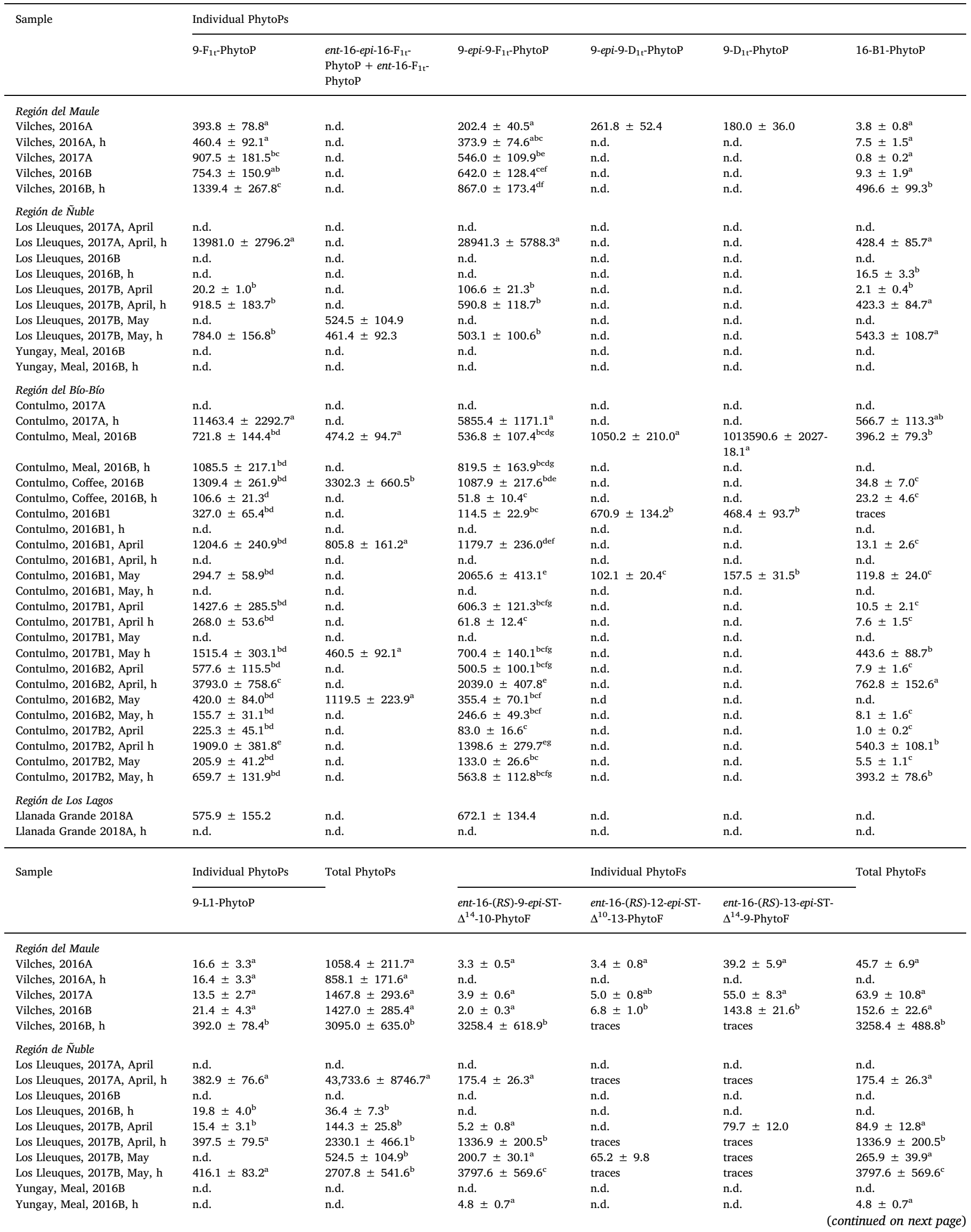


Table 3 (continued)

\begin{tabular}{|c|c|c|c|c|c|c|}
\hline \multirow[t]{2}{*}{ Sample } & \multirow{2}{*}{$\begin{array}{l}\text { Individual PhytoPs } \\
\text { 9-L1-PhytoP }\end{array}$} & \multirow[t]{2}{*}{ Total PhytoPs } & \multicolumn{3}{|c|}{ Individual PhytoFs } & \multirow[t]{2}{*}{ Total PhytoFs } \\
\hline & & & $\begin{array}{l}\text { ent-16-(RS)-9-epi-ST- } \\
\Delta^{14}-10-\mathrm{PhytoF}\end{array}$ & $\begin{array}{l}\text { ent-16-(RS)-12-epi-ST- } \\
\Delta^{10}-13-\mathrm{PhytoF}\end{array}$ & $\begin{array}{l}\text { ent-16-(RS)-13-epi-ST- } \\
\Delta^{14} \text {-9-PhytoF }\end{array}$ & \\
\hline \multicolumn{7}{|l|}{ Región del Bío-Bío } \\
\hline Contulmo, 2017A & n.d. & n.d. & $4.1 \pm 0.6^{\mathrm{a}}$ & n.d. & n.d. & $4.1 \pm 0.6 \mathrm{a}$ \\
\hline Contulmo, 2017A, h & $431.9 \pm 86.4^{\mathrm{a}}$ & $18,317.4 \pm 3663.5^{\mathrm{a}}$ & $1157.7 \pm 173.6^{\mathrm{b}}$ & traces & traces & $1157.7 \pm 173.6^{b}$ \\
\hline Contulmo, Meal, 2016B & $383.8 \pm 76.8^{\mathrm{a}}$ & $\begin{array}{l}1017153.6 \pm 2034- \\
30.7^{\mathrm{b}}\end{array}$ & $249.1 \pm 37.4^{\mathrm{ae}}$ & $118.0 \pm 17.7^{\mathrm{a}}$ & $30.3 \pm 4.6^{\mathrm{a}}$ & $397.5 \pm 59.6^{\mathrm{ef}}$ \\
\hline Contulmo, Meal, 2016B, h & n.d. & $1905.0 \pm 381.0^{\mathrm{a}}$ & $2221.7 \pm 316.3^{\mathrm{c}}$ & traces & traces & $2221.7 \pm 333.3^{c}$ \\
\hline Contulmo, Coffee, 2016B & $40.1 \pm 8.0^{\mathrm{b}}$ & $5774.5 \pm 1158.7^{\mathrm{a}}$ & $4.6 \pm 0.7^{\mathrm{a}}$ & $4.9 \pm 0.7^{b}$ & n.d. & $9.5 \pm 1.4^{\mathrm{a}}$ \\
\hline Contulmo, Coffee, 2016B, h & $23.9 \pm 4.8^{\mathrm{b}}$ & $205.5 \pm 41.1^{\mathrm{a}}$ & $3.9 \pm 0.6^{\mathrm{a}}$ & n.d. & $29.0 \pm 4.4^{\mathrm{a}}$ & $32.9 \pm 5.0^{\mathrm{a}}$ \\
\hline Contulmo, 2016B1 & $13.0 \pm 2.6^{\mathrm{b}}$ & $1593.9 \pm 318.8^{\mathrm{a}}$ & $0.8 \pm 0.1^{\mathrm{a}}$ & $44.0 \pm 6.6^{\mathrm{bc}}$ & $74.9 \pm 11.2^{\mathrm{b}}$ & $119.7 \pm 18.0^{\mathrm{e}}$ \\
\hline Contulmo, 2016B1, h & n.d. & n.d. & $9.1 \pm 1.4^{\mathrm{a}}$ & n.d. & n.d. & $9.1 \pm 1.4^{\mathrm{a}}$ \\
\hline Contulmo, 2016B1, April & $28.9 \pm 5.6^{b}$ & $3231.3 \pm 646.3^{\mathrm{a}}$ & $1.8 \pm 0.3^{\mathrm{a}}$ & n.d. & n.d. & $1.8 \pm 0.3^{\mathrm{a}}$ \\
\hline Contulmo, 2016B1, April, h & n.d. & n.d. & $1633.7 \pm 245.1^{\mathrm{d}}$ & $56.9 \pm 8.5^{c}$ & traces & $1690.6 \pm 253.6^{\mathrm{d}}$ \\
\hline Contulmo, 2016B1, May & $103.6 \pm 20.7^{b}$ & $2843.3 \pm 568.7^{\mathrm{a}}$ & $4.7 \pm 0.7^{\mathrm{a}}$ & $6.6 \pm 1.0^{\mathrm{b}}$ & $105.0 \pm 15.7^{c}$ & $116.2 \pm 17.4^{\mathrm{ae}}$ \\
\hline Contulmo, 2016B1, May, h & n.d. & n.d. & n.d. & n.d. & n.d. & n.d. \\
\hline Contulmo, 2017B1, April & $24.4 \pm 4.9^{\mathrm{b}}$ & $2068.8 \pm 413.8^{\mathrm{a}}$ & n.d. & n.d. & n.d. & n.d. \\
\hline Contulmo, 2017B1, April h & $16.6 \pm 3.2^{\mathrm{b}}$ & $354.0 \pm 70.8^{\mathrm{a}}$ & $248.2 \pm 37.2^{\mathrm{ae}}$ & $160.3 \pm 24.0^{\mathrm{ad}}$ & traces & $408.4 \pm 1.3^{\mathrm{e}}$ \\
\hline Contulmo, 2017B1, May & n.d. & n.d. & n.d. & n.d. & n.d. & n.d. \\
\hline Contulmo, 2017B1, May h & $426.6 \pm 85.3^{\mathrm{a}}$ & $3546.6 \pm 709.3^{\mathrm{a}}$ & n.d. & n.d. & n.d. & n.d. \\
\hline Contulmo, 2016B2, April & $19.1 \pm 3.8^{\mathrm{b}}$ & $1105.0 \pm 221.0^{\mathrm{a}}$ & $4.1 \pm 0.6^{\mathrm{a}}$ & n.d. & n.d. & $4.1 \pm 0.6^{\mathrm{a}}$ \\
\hline Contulmo, 2016B2, April, h & $634.1 \pm 126.8^{c}$ & $7228.8 \pm 1445.8^{\mathrm{a}}$ & $492.6 \pm 73.9^{\mathrm{ef}}$ & $182.2 \pm 27.3^{\mathrm{d}}$ & traces & $674.8 \pm 101.2^{\mathrm{fg}}$ \\
\hline Contulmo, 2016B2, May & n.d. & $1894.9 \pm 379.0^{\mathrm{a}}$ & $3.8 \pm 0.6^{\mathrm{a}}$ & n.d. & n.d. & $3.8 \pm 0.6^{\mathrm{a}}$ \\
\hline Contulmo, 2016B2, May, h & $18.5 \pm 3.7^{b}$ & $428.9 \pm 85.8^{\mathrm{a}}$ & $4.4 \pm 0.7^{\mathrm{a}}$ & n.d. & n.d. & $4.4 \pm 0.7^{\mathrm{a}}$ \\
\hline Contulmo, 2017B2, April & $13.6 \pm 2.7^{\mathrm{b}}$ & $322.9 \pm 64.6^{\mathrm{a}}$ & $3.4 \pm 0.5^{\mathrm{a}}$ & n.d. & n.d. & $3.4 \pm 0.5^{\mathrm{a}}$ \\
\hline Contulmo, 2017B2, April h & $438.3 \pm 87.7^{\mathrm{a}}$ & $4286.2 \pm 857.2^{\mathrm{a}}$ & $760.4 \pm 114.1^{\mathrm{f}}$ & traces & traces & $760.4 \pm 114.1^{g}$ \\
\hline Contulmo, 2017B2, May & $15.1 \pm 3.0^{\mathrm{b}}$ & $359.5 \pm 71.9^{\mathrm{a}}$ & $4.6 \pm 0.7^{\mathrm{a}}$ & n.d. & n.d. & $4.6 \pm 0.7^{\mathrm{a}}$ \\
\hline Contulmo, 2017B2, May, h & $379.4 \pm 35.5^{\mathrm{a}}$ & $1996.1 \pm 399.2^{\mathrm{a}}$ & traces & traces & traces & traces \\
\hline \multicolumn{7}{|l|}{ Región de Los Lagos } \\
\hline Llanada Grande 2018A & n.d. & $1248.0 \pm 249.6$ & traces & traces & traces & traces \\
\hline Llanada Grande 2018A, h & n.d. & n.d. & traces & traces & traces & traces \\
\hline
\end{tabular}

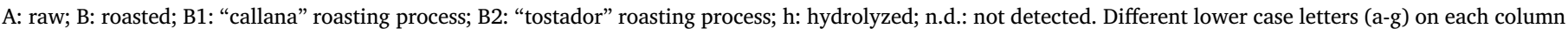
show significant differences according to Tukey's test $(p<0.05)$.

process of almonds induces changes in the TP content along with the temperature and roasting time (Lin et al., 2016).

\subsection{HPLC-DAD and HPLC-MS/MS analysis of phenolic compounds in G. avellana nuts}

The chemical profiles of the polyphenol enriched extracts (PEE) of hazelnuts (unprocessed, roasted and coffee substitute) were determined by HPLC-DAD and HPLC-MS/MS. The total ion chromatogram of raw and roasted samples are shown in Fig. S6. The tentative identification of the phenolic in the PEEs is summarized in Table 5. The identification of phenolic compounds is described in the subsequent paragraphs.

The caffeic acid derivatives $1,2,3,6,8,9$ and 10, were identified by the loss of a hexose (compounds 2 and $\mathbf{9}$ ), glucuronate (compound 1), dihexose (compound 3), a hexose and a pentose (compound 6), a hexose and acetate (compound 8) or a rutinoside (compound 10), from their $[\mathrm{M}-\mathrm{H}]^{-}$ions, leading to the $\mathrm{m} / \mathrm{z}$ ion at $178.9 \mathrm{amu}$, characteristic of caffeic acid. The compound 4 with a $[\mathrm{M}-\mathrm{H}]^{-}$ion at $m / z 254.90 \mathrm{amu}$ showed the loss of a phenyl unit, leading to $\mathrm{m} / \mathrm{z} 178.80 \mathrm{amu}$, in agreement with phenyl caffeate. A phenetyl caffeate hexoside (compound 11) and a pentoside (compound 15) were assigned based on the neutral loss of 162 and $132 \mathrm{amu}$, respectively, leading to the molecular ion at $\mathrm{m} / \mathrm{z} 268.8 \mathrm{amu}$ and further loss to $161 \mathrm{amu}$, in agreement with phenetyl caffeate glycosides. Compound 5 showed a $[\mathrm{M}-\mathrm{H}]^{-}$molecular ion at $m / z 121.55 \mathrm{amu}$, while compound 7 showed a $\mathrm{m} / \mathrm{z}$ molecular ion at $137.34 \mathrm{amu}$. Both compounds showed the loss of $\mathrm{CO}_{2}(-44 \mathrm{amu})$ and were identified as benzoic acid and hydroxybenzoic acid, respectively. The identity of these compounds was confirmed by co-injection with authentic standards.

Several ferulic/isoferulic acid derivatives were also tentatively identified. Compound 12 showed a $[\mathrm{M}-\mathrm{H}]^{-}$molecular ion at $\mathrm{m} / \mathrm{z}$
$501.61 \mathrm{amu}$, with a neutral loss of $308 \mathrm{amu}$, in agreement with rutinoside. The compound was identified as ferulic/isoferulic acid rhamnoside hexoside. Compound 16 showed a $[\mathrm{M}-\mathrm{H}]^{-}$molecular ion at $\mathrm{m} / \mathrm{z}$ $309.81 \mathrm{amu}$, and the same $\mathrm{MS}^{2}$ fragment ion that compound 12. The compound 16 was assigned as ferulic/isoferulic acid glutarate by the neutral loss of $116 \mathrm{amu}$. Compound 17 showed a [M-H] ${ }^{-}$molecular ion at $m / z 192.92 \mathrm{amu}$, that after decarboxylation led to the $\mathrm{m} / \mathrm{z}$ ion at $149 \mathrm{amu}$. The compound was tentatively identified as ferulic/isoferulic acid. The mass spectrum of compound 14 showed a $[\mathrm{M}-\mathrm{H}]^{-}$molecular ion at $385.63 \mathrm{amu}$, leading to the base peak at $\mathrm{m} / \mathrm{z} 222.99$ after the neutral loss of a hexose (162 amu). The compound was assigned as sinapic acid hexoside.

Quercetin dihexoside (compound 13) was identified by the $[\mathrm{M}-\mathrm{H}]^{-}$ molecular ion at $m / z 625.49$, leading to the $\mathrm{MS}^{2}$ fragment ion at 462.98 and $301.10 \mathrm{amu}$, after the neutral loss of two hexoses. The identity of the sugars and the relative placement in the aglycone remains to be established.

In a study on hazelnut (Corylus avellana, Betulaceae) kernels, oil and bagasse, Slatnar, Mikulic-Petkovsek, Stampar, Veberic, and Solat (2014) reported the occurrence of flavan-3-ol derivatives including procyanidin dimers, trimers, and tetramers, (epi)-catechin, flavonoid glycosides and hydrolysable tannins from Slovenian samples. Jakopic et al. (2011) described the phenolics occurring in the kernels of 20 hazelnut cultivars by HPLC-MS. The major phenolic compounds reported were also flavan-3-ol, procyanidins (dimers and trimers), flavonoids, gallic acid and protocatechuic acid. In our Chilean hazelnut samples, the composition is different than that reported for other nuts, with a very low phenolic content and the occurrence of simple phenolic acids, such as caffeic and ferulic/isoferulic acid derivatives. Condensed and hydrolysable tannins are usually the main phenolics in nuts (Chang et al., 2016). However, the tropical/subtropical Macadamia nuts and 
Table 4

Yields of phenolic-enriched extract (PEE) from the dry starting material, total phenolic content, antioxidant and inhibitory activity of the G. avellana PEEs towards $\alpha$ glucosidase.

\begin{tabular}{|c|c|c|c|c|c|c|c|}
\hline Sample & $\begin{array}{l}\text { PEE } \\
\%\end{array}$ & $\begin{array}{l}\text { TP } \\
\text { g GAE/100 g PEE }\end{array}$ & $\begin{array}{l}\text { FRAP } \\
\text { mmol TE/g PEE }\end{array}$ & $\begin{array}{l}\text { TEAC } \\
\mu \mathrm{M} \mathrm{TE} / \mathrm{g} \text { PEE }\end{array}$ & $\begin{array}{l}\text { DPPH } \\
\% \text { at } 100 \mu \mathrm{g} / \mathrm{mL} \text { oSC }_{50}(\mu \mathrm{g} / \\
\mathrm{mL})\end{array}$ & $\begin{array}{l}\text { ORAC } \\
\mu \mathrm{mol} \mathrm{TE} / \mathrm{g} \text { PEE }\end{array}$ & $\begin{array}{l}\alpha \text {-glucosidase inhibition } \\
\% \text { at } 100 \mu \mathrm{g} / \mathrm{mL} \text { or } \mathrm{IC}_{50}(\mu \mathrm{g} / \\
\mathrm{mL})\end{array}$ \\
\hline \multicolumn{8}{|l|}{ Región del Maule } \\
\hline Vilches, 2016A & 0.30 & $1.9 \pm 0.0^{\mathrm{a}}$ & $51.5 \pm 1.6^{\mathrm{a}}$ & inactive & $8.9 \pm 0.3 \%^{\mathrm{a}}$ & $954.4 \pm 21.2^{\mathrm{a}}$ & inactive \\
\hline Vilches, 2017A & 0.02 & $3.2 \pm 0.1^{\mathrm{b}}$ & $130.1 \pm 2.5^{\mathrm{b}}$ & inactive & inactive & $1380.3 \pm 32.6^{\mathrm{b}}$ & $31.4 \pm 1.6^{\mathrm{a}}$ \\
\hline Vilches, 2016B & 0.85 & $2.1 \pm 0.1^{\mathrm{a}}$ & $139.0 \pm 11.8^{\mathrm{b}}$ & inactive & $14.8 \pm 1.3 \%^{\mathrm{b}}$ & $374.5 \pm 10.6^{\mathrm{c}}$ & $24.8 \pm 0.7^{\mathrm{b}}$ \\
\hline \multicolumn{8}{|l|}{ Región de Ñuble } \\
\hline Los Lleques, 2017A, April & 0.02 & $*$ & $110.4 \pm 5.5^{\mathrm{a}}$ & $207.3 \pm 4.3^{\mathrm{a}}$ & $26.1 \pm 1.2 \%^{\mathrm{b}}$ & $1173.9 \pm 6.5^{\mathrm{c}}$ & $10.8 \pm 0.9^{\mathrm{a}}$ \\
\hline Los Lleuques, 2017A, May & 0.03 & $4.5 \pm 0.1^{\mathrm{a}}$ & $242.0 \pm 4.4^{\mathrm{b}}$ & $260.2 \pm 5.4^{\mathrm{d}}$ & $38.1 \pm 2.0 \%^{\mathrm{c}}$ & $1188.7 \pm 31.9^{c}$ & inactive \\
\hline Los Lleuques, 2016B & 0.22 & $1.6 \pm 0.0^{\mathrm{b}}$ & $46.2 \pm 4.6^{c}$ & inactive & $15.5 \pm 0.3 \%^{\mathrm{d}}$ & $273.9 \pm 7.7^{\mathrm{d}}$ & $79.7 \pm 2.6^{\mathrm{b}}$ \\
\hline Los Lleques, 2017B, April & 0.03 & $*$ & $208.2 \pm 3.8^{\mathrm{d}}$ & $257.8 \pm 5.3^{\mathrm{d}}$ & $42.2 \pm 0.7 \%^{\mathrm{e}}$ & $1186.6 \pm 3.5^{c}$ & $17.5 \pm 0.4^{\mathrm{a}}$ \\
\hline Los Lleques, 2017B, May & 0.01 & $7.6 \pm 0.2^{c}$ & $592.4 \pm 10.5^{\mathrm{e}}$ & $782.8 \pm 16.3^{b}$ & $65.9 \pm 2.2^{\mathrm{a}}$ & $956.4 \pm 48.4^{\mathrm{a}}$ & inactive \\
\hline Los Lleuques, Meal 2016B & 0.19 & $3.0 \pm 0.0^{\mathrm{d}}$ & $148.0 \pm 7.9^{\mathrm{f}}$ & inactive & $31.4 \pm 1.1 \%{ }^{\mathrm{f}}$ & $1225.0 \pm 40.1^{c}$ & $66.5 \pm 5.3^{c}$ \\
\hline Yungay, Meal 2016B & 0.02 & $8.3 \pm 0.1^{\mathrm{e}}$ & $439.6 \pm 11.8^{g}$ & $627.6 \pm 20.5^{c}$ & $65.1 \pm 1.3^{\mathrm{a}}$ & $2157.5 \pm 64.2^{\mathrm{b}}$ & $12.3 \pm 0.7^{\mathrm{a}}$ \\
\hline \multicolumn{8}{|l|}{ Región del Bío-Bío } \\
\hline Contulmo, 2017A & 0.05 & $4.6 \pm 0.1^{\mathrm{a}, \mathrm{b}, \mathrm{c}, \mathrm{d}}$ & $352.8 \pm 11.8^{\mathrm{a}}$ & inactive & $42.6 \pm 1.3 \%^{\mathrm{a}}$ & $1569.7 \pm 66.0^{\mathrm{a}}$ & inactive \\
\hline Contulmo, Meal 2016B & 0.40 & $2.1 \pm 0.0^{\mathrm{e}}$ & inactive & inactive & $26.1 \pm 0.0 \%^{\mathrm{b}}$ & $421.0 \pm 9.6^{\mathrm{b}}$ & inactive \\
\hline Contulmo, Coffee 2016B & 0.23 & $8.7 \pm 0.1^{\mathrm{f}}$ & $559.7 \pm 13.7^{b}$ & inactive & $52.3 \pm 0.8^{\mathrm{d}}$ & $1935.7 \pm 47.8^{\mathrm{c,d}}$ & inactive \\
\hline Cotulmo, 2016B1 & 1.04 & $3.4 \pm 0.1^{\mathrm{b}, \mathrm{e}}$ & $898.9 \pm 18.0^{c}$ & $458.5 \pm 35.0^{\mathrm{a}}$ & $23.6 \pm 0.3 \%^{\mathrm{c}}$ & $1724.8 \pm 78.3^{\mathrm{a}, \mathrm{c}}$ & $37.6 \pm 2.6^{\mathrm{a}}$ \\
\hline Contulmo, 2016B1, April & 0.02 & $5.3 \pm 0.1^{\mathrm{c}, g}$ & $953.8 \pm 11.2^{\mathrm{d}}$ & $1012.8 \pm 7.0^{\mathrm{b}}$ & $70.8 \pm 2.2^{\mathrm{e}}$ & $2058.3 \pm 126.8^{\mathrm{d}, \mathrm{e}}$ & $5.0 \pm 0.4^{\mathrm{b}, \mathrm{c}}$ \\
\hline Contulmo, 2016B1, May & 0.02 & $6.6 \pm 0.1^{\mathrm{g}, \mathrm{h}, \mathrm{i}, \mathrm{j}, \mathrm{k}, \mathrm{l}}$ & $362.9 \pm 7.6^{\mathrm{a}}$ & $499.4 \pm 14.4^{\mathrm{a}, \mathrm{c}}$ & $93.8 \pm 1.6^{\mathrm{f}}$ & $1920.9 \pm 168.4^{\mathrm{c}, \mathrm{e}, \mathrm{f}}$ & $*$ \\
\hline Contulmo, 2017B1, April & 0.01 & $4.5 \pm 0.1^{\mathrm{a}, \mathrm{b}, \mathrm{c}, \mathrm{d}}$ & $673.9 \pm 7.0 \mathrm{e}$ & $711.9 \pm 3.0^{\mathrm{d}}$ & $73.5 \pm 0.4^{\mathrm{e}, \mathrm{g}}$ & $1665.7 \pm 126.5^{\mathrm{a}, \mathrm{f}}$ & $8.5 \pm 0.6^{\mathrm{d}}$ \\
\hline Contulmo, 2017B1, May & 0.02 & $7.2 \pm 0.2^{\mathrm{f}, \mathrm{h}, \mathrm{m}}$ & $384.6 \pm 23.3^{\mathrm{a}}$ & $678.6 \pm 22.9^{d}$ & $77.6 \pm 1.5^{\mathrm{g}, \mathrm{h}}$ & $1232.8 \pm 3.2^{\mathrm{g}}$ & $7.9 \pm 0.4^{\mathrm{d}}$ \\
\hline Contulmo, 2016B2, April & 0.02 & $7.0 \pm 0.2^{\mathrm{i}, \mathrm{m}}$ & $429.4 \pm 9.5^{\mathrm{f}}$ & $548.5 \pm 7.0^{\mathrm{c}}$ & $75.3 \pm 2.0^{\mathrm{e}, \mathrm{h}}$ & $863.1 \pm 18.8^{\mathrm{h}}$ & $87.4 \pm 0.6^{\mathrm{e}}$ \\
\hline Contulmo, 2016B2, May & 0.03 & $5.2 \pm 0.1^{\mathrm{d}, \mathrm{j}}$ & $778.4 \pm 15.1^{g}$ & $732.9 \pm 3.1^{\mathrm{d}}$ & $62.0 \pm 0.6^{\mathrm{i}}$ & $1593.5 \pm 91.7^{\mathrm{a}}$ & $76.4 \pm 0.5^{f}$ \\
\hline Contulmo, 2017B2, April & 0.03 & $8.6 \pm 0.2^{\mathrm{f}, \mathrm{k}, \mathrm{m}}$ & $642.9 \pm 16.4^{\mathrm{e}}$ & $944.1 \pm 31.0^{\mathrm{e}}$ & $50.5 \pm 1.4^{\mathrm{d}}$ & $996.9 \pm 83.7^{\mathrm{g}, \mathrm{h}}$ & $3.7 \pm 0.3^{\mathrm{b}}$ \\
\hline Contulmo, 2017B2, May & 0.01 & $7.5 \pm 0.1^{\mathrm{f}, 1, \mathrm{~m}}$ & $474.7 \pm 18.1^{\mathrm{h}}$ & inactive & $85.0 \pm 3.0^{j}$ & $1051.3 \pm 92.6^{\mathrm{g}, \mathrm{h}}$ & $6.6 \pm 0.3^{\mathrm{c}, \mathrm{d}}$ \\
\hline \multicolumn{8}{|l|}{ Región de Los Lagos } \\
\hline Llanada Grande, 2018A & * & $2.70 \pm 0.04$ & $90.38 \pm 0.72$ & $506.07 \pm 14.81$ & $14.0 \pm 0.3 \%$ & inactive & $60.9 \pm 1.1 \%$ \\
\hline Quercetin $\#$ & - & - & $1077.2 \pm 16.4$ & $8157.9 \pm 22.1$ & $7.8 \pm 0.3$ & $22561.6 \pm 808.8$ & - \\
\hline Acarbose $^{\#}$ & - & - & - & - & - & - & $120.9 \pm 2.0$ \\
\hline
\end{tabular}

A: raw; B: roasted; B1 "callana" roasting process; B2: "tostador" roasting process; PEE: polyphenol-enriched extract; TP: total phenolic content; FRAP: ferric reducing antioxidant power; TEAC: trolox equivalents antioxidant capacity; DPPH: 2,2-diphenyl-1-picrylhydrazyl radical; ORAC: oxygen radical antioxidant capacity. Different lower case letters (a-m) in the same column show significant differences within each region, according to Tukey's test $(p<0.05)$; *not determined due to low amount of material. ${ }^{\#}$ reference compounds.

the Chilean relative G. avellana show low content of phenolics. Following the trend observed for pecan and macadamia nuts, flavan-3-ols such as catechin and (epi)-catechin were not detected in the G. avellana cotyledons or its commercial products, neither procyanidins nor proanthocyanidins. Chang et al. (2016) reported the presence of hydroxybenzoic and hydroxycinnamic acids in some nuts, showing that these compounds are less common than flavan 3-ol derivatives. According to these authors, gallic acid has not been detected in almonds and macadamia nuts. The hexosides of syringic and $p$-coumaric acid occur in the hazelnut pellet. In addition, the occurrence of 2,6-

Table 5

Tentative identification of G. avellana phenolics by HPLC-MS-MS in the negative ion mode.

\begin{tabular}{|c|c|c|c|c|}
\hline Compound & Rt (min) & {$[\mathrm{M}-\mathrm{H}]^{-}$} & MS-MS & Tentative identification \\
\hline 1 & 3.4 & 355.53 & $322.99\left(\begin{array}{lll}1 & 0 & 0\end{array}\right), 179.14(57)$ & Caffeic acid glucuronate \\
\hline 2 & $4.0-4.2$ & 341.03 & $178.80(100), 143.20(25), 119.00(28)$ & Caffeic acid hexoside \\
\hline 3 & 5.8 & 503.26 & 414.50 (50), 340.70 (36), 332.80 (36), 220.50 (29), 178.90 (1 00 ) & Caffeic acid dihexoside \\
\hline 4 & $7.2-7-4$ & 254.90 & $178.80\left(\begin{array}{lll}1 & 0 & 0\end{array}\right)$ & Phenyl caffeate \\
\hline 5 & 12.5 & 121.55 & 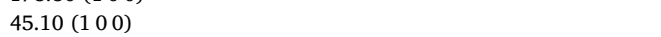 & Benzoic acid* \\
\hline 6 & 12.8 & 473.21 & $340.20(41), 178.83\left(\begin{array}{lll}1 & 0 & 0\end{array}\right)$ & Caffeic acid hexoside pentoside \\
\hline 7 & 15.3 & 137.34 & $92.90\left(\begin{array}{llllllllll}1 & 0 & 0\end{array}\right)$ & Hydroxybenzoic acid* \\
\hline 8 & 17.8 & 401.27 & 238.91 (1 00 ), 178.97 (50) & Caffeic acid acetate hexoside \\
\hline 9 & 19.4 & 341.82 & $296.80(100), 178.60(38)$ & Caffeic acid hexoside isomer \\
\hline 10 & 23.3 & 487.83 & $178.85\left(\begin{array}{lllllllll}1 & 0 & 0\end{array}\right)$ & Caffeic acid rhamnoside hexoside (rutinoside) \\
\hline 11 & 23.5 & 431.73 & $268.82(100), 160.85(57)$ & Phenetyl caffeate hexoside \\
\hline 12 & 23.9 & 501.61 & $192.89\left(\begin{array}{lllllllll}1 & 0 & 0\end{array}\right)$ & Ferulic/isoferulic acid rhamnoside hexoside \\
\hline 13 & 28.2 & 625.49 & 462.98 ( 100$), 301.10(16)$ & Quercetin dihexoside \\
\hline 14 & 28.4 & 385.63 & $222.99\left(\begin{array}{llll}1 & 0 & 0\end{array}\right)$ & Sinapic acid hexoside \\
\hline 15 & $28.6-28.7$ & 401.44 & $268.91(100), 161.10(12)$ & Phenetyl caffeate pentoside \\
\hline 16 & $31.9-32.1$ & 309.81 & $192.97\left(\begin{array}{lll}1 & 0 & 0\end{array}\right)$ & Ferulic/isoferulic acid glutarate \\
\hline 17 & $36.3-36.5$ & 192.92 & 149.01 & Ferulic/isoferulic acid \\
\hline
\end{tabular}

HPLC-MS-MS: high performance liquid chromatography coupled to mass spectrometry. Rt: retention time;

$[\mathrm{M}-\mathrm{H}]^{-}$: molecular ion; MS-MS: molecular fragment ion. "Identity confirmed by co-injection with authentic standards. 
diydroxybenzoic acid and 3,5-dimethoxy-4-hydroxycinnamic acid in the hard shell of macadamia has been reported (Chang et al., 2016). In G. avellana as well as in other nuts, the husk is removed before roasting, leading to the loss of bioactive compounds. This effect was recently described by Taş and Gökmen (2017) in the skin and cotyledons of several nuts.

\subsection{6. NMR analysis of the polyphenol-enriched extracts (PEEs) from G. avellana nuts}

In the PEE from the coffee substitute and the roasted cotyledons, ${ }^{1} \mathrm{H}$ NMR analysis showed a main compound with a s at $\delta 9.59(1 \mathrm{H}), 7.40 \mathrm{~d}$ $(3.2 \mathrm{~Hz} ; 1 \mathrm{H}), 6.60 \mathrm{~d}(3.2 \mathrm{~Hz} ; 1 \mathrm{H})$ and $4.63 \mathrm{~s}(2 \mathrm{H})$, in agreement with 5hydroxymethylfurfural (5-HMF). The identity of this compound was confirmed by comparison of the Rt and UV spectrum with that of a reference standard. Since 5-HMF is absent in raw cotyledons, its presence indicates that the compound is produced during roasting. Aromatic signals at $\delta 7.88 \mathrm{~d}(8.8)$ and $6.83 \mathrm{~d}(8.8)$, support the occurrence of a $p$-substituted aromatic ring compatible with $p$-hydroxybenzoic acid. The identity of this compound was confirmed by comparison with a standard sample, matching in Rt and UV spectrum of compound 7. In addition, when the cotyledons were processed to afford the PEE, a colorless solid crystalized and it was identified as the disaccharide sucrose by the ${ }^{1} \mathrm{H}$ and ${ }^{13} \mathrm{C}$ NMR data, in agreement with De Bruyn and van Loo (1991).

\subsection{Antioxidant activity of the polyphenol-enriched extracts (PEEs) from G. avellana nuts}

The antioxidant activity of the PEEs was assessed using different techniques including FRAP (mmol TE/g PEE), TEAC ( $\mu \mathrm{M} \mathrm{TE/g} \mathrm{PEE),}$ DPPH (\% discoloration at $100 \mu \mathrm{g} / \mathrm{mL}$ or $\mathrm{SC}_{50}$ in $\mu \mathrm{g} / \mathrm{mL}$ ), and ORAC ( $\mu \mathrm{mol} \mathrm{TE} / \mathrm{g}$ PEE) (Table 4).

Regarding the FRAP assay, the raw cotyledons showed values ranging from 51.5 to $352.8 \mathrm{mmol} \mathrm{TE} / \mathrm{g}$ PEE. The roasted samples presented values ranging from 46.2 to $953.8 \mathrm{mmol} \mathrm{TE} / \mathrm{g}$ PEE. The coffee substitute showed a value of $559.7 \mathrm{mmol} \mathrm{TE} / \mathrm{g}$ PEE, while only one of the meal samples was active, with a value of $439.6 \mathrm{mmol} \mathrm{TE} / \mathrm{g}$ PEE. In the TEAC assay, the results for raw samples ranged from inactive to $506.1 \mu \mathrm{M}$ TE/g PEE. The roasted samples showed values ranging from inactive to $944.1 \mu \mathrm{M}$ TE/g PEE. The coffee and one of the meal samples proved to be inactive, while the meal sample from Región de Nuble showed a value of $627.6 \mu \mathrm{M} \mathrm{TE} / \mathrm{g}$ PEE. In the DPPH assay, the most active samples were the roasted collections from Región del Bío-Bío, with $\mathrm{SC}_{50}$ values ranging from 50.5 to $93.8 \mu \mathrm{g} / \mathrm{mL}$. The most active samples in the ORAC assay were the meal sample from Región de Nuble (2157.5 $\mu \mathrm{mol} \mathrm{TE} / \mathrm{g}$ extract), the coffee substitute sample from Región del Bío-Bío (1935.7 $\mu \mathrm{mol} \mathrm{TE/g} \mathrm{extract)} \mathrm{and} \mathrm{the} \mathrm{samples} \mathrm{from} \mathrm{Contulmo}$ roasted by means of "callana" process during 2016 (2058.3 and $1920.9 \mu \mathrm{mol} \mathrm{TE} / \mathrm{g}$ extract, respectively). Moure et al. (2000) investigated the antioxidant activity of extracts obtained from the nonedible hulls from $G$. avellana, but not from the cotyledons. The polarity and composition of the hull extracts is very different from that of the extracts investigated in our study. The report by Ebrahem, Richardson, and Tetley (1994) refer mainly to the oils of a different species, namely Corylus avellana (European hazelnut). In the recent study by Flores García et al. (2018), the authors partitioned the oil with MeOH:water 60:40 and looked for antioxidant activity in the polar phase by means of the DPPH discoloration assay. The authors reported that the G. avellana oil extract scavenged the radical by less than $40 \%$, being $44 \%$ less active than olive oil. The overall antioxidant activity of our samples can be considered as low when compared to other fruits, in agreement with the low total phenolic content. Interestingly, it has been reported that the roasting process decreases the antioxidant activity of almonds, Brazilian nuts, and cashew nuts by means of DPPH assay (Taș \& Gökmen, 2017), while in our samples, the roasting process increased the discoloration of the DPPH radical.

\subsection{Enzyme inhibition assays}

3.8.1. Inhibition of metabolic syndrome-associated enzymes by the PEEs from $G$. avellana

It has been reported that consumption of nuts is associated with an improvement in the conditions of metabolic syndrome (Kendall, Josse, Esfahani, \& Jenkins, 2010). The inhibition of the enzymes $\alpha$-amylase, $\alpha$ glucosidase, and pancreatic lipase by the G. avellana PEEs was assessed. Most of the PEEs were inactive towards $\alpha$-amylase at $100 \mu \mathrm{g} / \mathrm{mL}$ (data not shown). Three samples showed weak activity $(<10 \%)$, while only the coffee substitute showed inhibitory activity by $12.5 \%$ at $100 \mu \mathrm{g} / \mathrm{mL}$. Under the same experimental conditions, the positive control acarbose showed an $\mathrm{IC}_{50}$ value of $28.5 \mu \mathrm{g} / \mathrm{mL}$. The effect for all samples should be regarded as not relevant against $\alpha$-amylase. In the same way, all the samples were inactive against pancreatic lipase at $50 \mu \mathrm{g} / \mathrm{mL}$ (data not shown) while the positive control orlistat showed an $\mathrm{IC}_{50}$ value of $0.04 \mu \mathrm{g} / \mathrm{mL}$. Interestingly, the samples showed inhibitory effect against $\alpha$-glucosidase (Table 4), with $\mathrm{IC}_{50}$ values ranging from 5.0 to $87.4 \mu \mathrm{g}$ / $\mathrm{mL}$. The acarbose control showed an $\mathrm{IC}_{50}$ value of $120 \mu \mathrm{g} / \mathrm{mL}$. No clear tendency was observed between raw and roasted samples, in terms of inhibitory activity towards this enzyme. Studies carried out with humans have shown that nuts can diminish the postprandial glycaemic response when consumed together with high-glycaemic index foods in normo-glycemic as well as type- 2 diabetic patients (Kendall et al., 2010)

3.8.2. Inhibition of pro-inflammatory enzymes by oils from $G$. avellana nuts

The oils obtained from $G$. avellana samples were evaluated for their inhibitory effect towards the enzymes COX-1, COX-2 and LOX. Seven selected samples were assessed at $100 \mu \mathrm{g} / \mathrm{mL}$, and included oils from raw and roasted cotyledons (Table 2). The most active oil against COX1 was that from the raw cotyledons collected at Los Lleuques $(79.9 \%$ inhibition at $100 \mu \mathrm{g} / \mathrm{mL}$ ) followed by the raw and the "tostador" roasted cotyledon oil from Contulmo (73.1 and 71.8\%, respectively). The effect on COX-2 was less pronounced, with some selectivity for the roasted cotyledon oil from Vilches and Los Lleuques, which were inactive against COX-1, but inhibited COX-2 by 33.8 and $20.6 \%$, respectively. The roasting process induced a loss in the inhibitory activity against COX-1. The Pearson's coefficient showed a significant positive correlation between the C18:0 content and the inhibition of COX-2 $(\mathrm{r}=0.728, p<0.05)$.

The selected oil samples inhibited LOX in the range of 9.1-26.0\%. The roasting process significantly increased the percentage of inhibition against LOX in the samples collected at Región del Maule and Región de Ñuble (Table 2). Our results are in agreement with previous studies reporting that fatty acids display anti-inflammatory effect. This can be associated with the oil composition (Calder, 2005; Das, 2008). The Pearson's coefficient showed positive correlations between the LOX inhibition and the content of $\mathrm{C} 20: 0(\mathrm{r}=0.558, p<0.01)$ and $\mathrm{C} 21: 0$ $(\mathrm{r}=0.487, p<0.05)$. Oleic acid, one of the main fatty acid in the $G$. avellana oils, has shown mild inhibition against COX-1 in vitro (Henry, Momin, Nair, \& Dewitt, 2002); however, no significant correlation was detected in our samples. Kohatsu and Karpowicz (2018) reviewed the relation of diet and inflammation, associating the composition of the Mediterranean diet with the anti-inflammatory effect of their components. Fats are relevant in the Mediterranean diet and are mainly plant oils with high content of the essential fatty acids omega- 6 (linoleic acid) and omega-3 ( $\alpha$-linolenic acid). In the 23 oil samples from G. avellana, the linoleic acid content ranged between $42-70.4 \mathrm{~g} / \mathrm{L}$ (Table 1 ).

\section{Conclusions}

Twenty three samples from $G$. avellana cotyledons were studied to assess the content and composition of phytochemicals before and after 
the roasting process. In general, the phenolic content was low and, consequently, the antioxidant activity of the samples was moderate to low. Seventeen compounds were detected in the PEEs from the samples, most of them derived from caffeic, benzoic and ferulic/isoferulic acids. Oleic and 7-hexadecenoic acids were the main fatty acids quantified. When the selected oil samples were submitted to UV radiation in the presence of a spin trap (EPR), a strong radical scavenging capacity was observed. Eight PhytoPs and three PhytoFs were detected and quantified for the first time in G. avellana cotyledons. The main PhytoPs were 9- $\mathrm{F}_{1 \mathrm{t}}$-PhytoP and 9-epi-9- $\mathrm{F}_{1 \mathrm{t}}$-PhytoP, while ent-16-(RS)-9-epi-ST- $\Delta^{14}-10$ PhytoF was the main PhytoF. Moderate inhibitory activity against $\alpha$ glucosidase was displayed by the PEEs. A mild effect was observed in the inhibition of pro-inflammatory enzymes by the selected oil samples. The absence of clearly differentiated clusters in the PCA analysis indicates that there were no differences neither by raw and roasted samples, nor by the geographical origin of the studied material. Gevuina avellana nuts are a source of fatty acids, oxylipins and polyphenols with potential health promoting activities, confirming our hypothesis.

\section{Declaration of Competing Interest}

None.

\section{Acknowledgements}

Financial support from FONDECYT 1170090 (Chile) is acknowledged. LLPE thanks the Universidad de Talca (Chile) for a Doctoral grant. AB-E thanks CONICYT (Chile) for his doctoral grant 211515612015. This work was partially funded by the "Fundación Séneca de la Región de Murcia" Grupo de Excelencia 19900/GERM/15, the Spanish Research Council (CEBAS-CSIC) and the CNRS, by "Projet International de Coopération Scientifique" (PICS-2015-261141) and the Spanish project AGL2017-83386-R from the Spanish Ministry of Science, Innovation and Universities. RDP was sponsored by a Postdoctoral Contract (Juan de la Cierva de Incorporación ICJI-2015-25373) from the Ministry of Economy, Industry and Competitiveness of Spain, respectively.

\section{Appendix A. Supplementary data}

Supplementary data to this article can be found online at https:// doi.org/10.1016/j.foodchem.2019.125026.

\section{References}

Birch, J., Yap, K., \& Silcock, P. (2010). International Journal of Food Science \& Technology, 45, 81-86. https://doi.org/10.1111/j.1365-2621.2009.02106.x.

Burgos-Edwards, A., Jiménez-Aspee, F., Thomas-Valdés, S., Schmeda-Hirschmann, G., \& Theoduloz, C. (2017). Qualitative and quantitative changes in polyphenol composition and bioactivity of Ribes magellanicum and $R$. punctatum after in vitro gastrointestinal digestion. Food Chemistry, 237, 1073-1082. https://doi.org/10.1016/j. foodchem.2017.06.060

Calder, P. C. (2005). Polyunsaturated fatty acids and inflammation. Biochemical Society Transactions, 33(423), 427. https://doi.org/10.1042/BST0330423.

Carrasco-Del Amor, A. M., Aguayo, E., Collado-Gonzalez, J., Guy, A., Galano, J.-M., Durand, T., \& Gil-Izquierdo, A. (2017). Impact of processing conditions on the phytoprostanes profile of three types of nut kernels. Free Radical Research, 51, 141-147. https://doi.org/10.1080/10715762.2017.1288909.

Chang, S. K., Alasalvar, C., Bolling, B. W., \& Shahidi, F. (2016). Nuts and their co-products: The impact of processing (roasting) on phenolics, bioavailability, and health benefits - A - comprehensive review. Journal of Functional Foods, 26, 88-122. https:// doi.org/10.1016/j.jff.2016.06.029.

Collado-Gonzalez, J., Medina, S., Durand, T., Guy, A., Galano, J. M., Torrecillas, A., ... GilIzquierdo, A. (2015). New UHPLC-QqQ-MS/MS method for quantitative and qualitative determination of free phytoprostanes in foodstuffs of commercial olive and sunflower oils. Food Chemistry, 178, 212-220. https://doi.org/10.1016/j.foodchem. 2015.01.097.

Cuyamandous, C., Leung, K. S., Durand, T., Lee, J. C. Y., Oger, C., \& Galano, J. M. (2015). Synthesis and discovery of phytofurans: Metabolites of [small alpha]-linolenic acid peroxidation. Chemical Communications, 51, 15696-15699. https://doi.org/10.1039/ C5CC05736A.

Cuyamendous, C. L., Leung, K. S., Bultel-Poncé, V., Guy, A., Durand, T., Galano, J. M., ...
Oger, C. (2017). Total synthesis and in vivo quantitation of phytofurans derived from $\alpha$-linolenic acid. European Journal of Organic Chemistry, 2486-2490. https://doi.org/ 10.1002/ejoc.201700270.

Das, U. N. (2008). Essential fatty acids and their metabolites could function as endogenous HMG-CoA reductase and ACE enzyme inhibitors, anti-arrhythmic, antihypertensive, anti-atherosclerotic, anti-inflammatory, cytoprotective, and cardioprotective molecules. Lipids in Health and Disease, 7, 1-18. https://doi.org/10.1186/ 1476-511X-7-37.

Davies, M. J. (1987). Applications of electron spin resonance spectroscopy to the identification of radicals produced during lipid peroxidation. Chemistry and Physics of Lipids, 44(2-4), 149-173.

De Bruyn, A., \& van Loo, J. (1991). The identification by ${ }^{1} \mathrm{H}$ and ${ }^{13} \mathrm{C}$-n.m.r. spectroscopy of sucrose, 1-kestose, and neokestose in mixtures present in plant extracts. Carbohydrate Research, 211(1), 131-136.

Durand, T., Bultel-Poncé, V., Guy, A., El Fangour, S., Rossi, J. C., \& Galano, J. M. (2011). Isoprostanes and phytoprostanes: Bioactive lipids. Biochimie, 93, 52-60. https://doi. org/10.1016/j.biochi.2010.05.014.

Ebrahem, K. S., Richardson, D. G., \& Tetley, R. M. (1994). Changes in oil content, fatty acid, and vitamin e composition in developing hazelnut kernels. Acta Horticulturae, 351, 669-676. https://doi.org/10.17660/ActaHortic. 1994.351.74.

El Fangour, S., Guy, A., Despres, V., Vidal, J. P., Rossi, J. C., \& Durand, T. (2004). Total synthesis of the eight diastereomers of the syn-anti-syn phytoprostanes F1 types I and II. Journal of Organic Chemistry, 69, 2498-2503. https://doi.org/10.1021/jo035638i.

Flores García, M., Vergara, C. E., Forero-Doria, O., Guzmán, L., \& Perez-Camino, M. del C. (2018). Chemical evaluation and thermal behavior of Chilean hazelnut oil (Gevuina avellana Mol.) a comparative study with extra virgin olive oil. European Food Research and Technology. https://doi.org/10.1007/s00217-018-3206-1.

Ghafoor, K., Özcan, M. M., AL-Juhaimi, F., Babiker, E. E., \& Fadimu, G. J. (2019). Changes in quality, bioactive compounds, fatty acids, tocopherols, and phenolic composition in oven- and microwave-roasted poppy seeds and oil. LWT-Food Science and Technology, 99, 490-496. https://doi.org/10.1016/j.lwt.2018.10.017.

Gray, J. (2005). Nuts and seeds. In B. Caballero, L. Allen, \& A. Prentice (Eds.). Encyclopedia of Human Nutrition (pp. 329-335). New York: Academic press.

Guy, A., Flanagan, S., Durand, T., Oger, C., \& Galano, J. M. (2015). Facile synthesis of cyclopentenone B1- and L1-type phytoprostanes. Frontiers in Chemistry, 3, 41. https:// doi.org/10.3389/fchem.2015.00041.

Heldrich, K. (2000). Official Methods of Analysis of the Association of Official Analytical Chemists. Arlington, VA: Association of Official Chemists.

Henry, G. E., Momin, R. A., Nair, M. G., \& Dewitt, D. L. (2002). Antioxidant and cyclooxygenase activities of fatty acids found in food. Journal of Agricultural and Food Chemistry, 50(8), 2231-2234.

Jakopic, J., Petkovsek, M. M., Likozar, A., Solar, A., Stampar, F., \& Veberic, R. (2011). HPLC-MS identification of phenols in hazelnut (Corylus avellana L.) kernels. Food Chemistry, 124, 1100-1106. https://doi.org/10.1016/j.foodchem.2010.06.011.

Jiménez-Aspee, F., Theoduloz, C., Gómez-Alonso, S., Hermosín-Gutiérrez, I., Reyes, M., \& Schmeda-Hirschmann, G. (2018). Polyphenolic profile and antioxidant activity of meristems and leaves from "chagual" (Puya chilensis Mol.), a salad from central Chile. Food Research International, 114, 90-96. https://doi.org/10.1016/j.foodres.2018.07. 051.

Kendall, C. W., Josse, A. R., Esfahani, A., \& Jenkins, D. J. (2010). Nuts, metabolic syndrome and diabetes. British Journal of Nutrition, 104, 465-473. https://doi.org/10. 1017/S0007114510001546.

Kohatsu, Wendy, \& Karpowicz, Scott (2018). Integrative Medicine (pp. 869-877.e4). Elsevier. https://doi.org/10.1016/B978-0-323-35868-2.00088-8.

León-Perez, D., Medina, S., Londoño-Londoño, J., Cano-Lamadrid, M., CarbonellBarrachina, A., Durand, T., ... Gil-Izquierdo, A. (2019). Comparative study of different cocoa (Theobroma cacao L.) clones in terms of their phytoprostanes and phytofurans contents. Food Chemistry, 280, 231-239. https://doi.org/10.1016/j. foodchem.2018.12.072

Lin, J. T., Liu, S.-C., Hu, C.-C., Shyu, Y.-S., Hsu, C.-Y., \& Yang, D.-J. (2016). Effects of roasting temperature and duration on fatty acid composition, phenolic composition, Maillard reaction degree and antioxidant attribute of almond (Prunus dulcis) kernel. Food Chemistry, 190, 520-528. https://doi.org/10.1016/j.foodchem.2015.06.004.

Loeffler, C., Berger, S., Guy, A., Durand, T., Bringmann, G., Dreyer, M., ... Mueller, M. J. (2005). B1-phytoprostanes trigger plant defense and detoxification responses. Plant Physiology, 137, 328-340. https://doi.org/10.1104/pp.104.051714.

Mösbach, E. W. (1992). Botánica Indígena de Chile. Santiago de Chile: Fundación Andes y Editorial Andrés Bello.

Moure, A., Franco, D., Sineiro, J., Domínguez, H., \& Núñez, M. J. (2003). Simulation of multistage extraction of antioxidants from Chilean hazelnut (Gevuina avellana) hulls. Journal of the American Oil Chemists Society, 80(4), 389-396.

Papadimitriou, V., Sotiroudis, T. G., Xenakis, A., Sofikiti, N., Stavyiannoudaki, V., \& Chaniotakis, N. A. (2006). Oxidative stability and radical scavenging activity of extra virgin olive oils: An electron paramagnetic resonance spectroscopy study. Analytica Chimica Acta, 573, 453-458. https://doi.org/10.1016/j.aca.2006.02.007.

Polovka, M. (2006). EPR spectroscopy: A tool to characterize stability and antioxidant properties of foods. Journal of Food and Nutrition Research, 45(1), 1-11.

Slatnar, A., Mikulic-Petkovsek, M., Stampar, F., Veberic, R., \& Solat, A. (2014). HPLC-MS ${ }^{\mathrm{n}}$ identification and quantification of phenolic compounds in hazelnut kernels, oil and bagasse pellets. Food Research International, 64, 783-789. https://doi.org/10.1016/j. foodres.2014.08.009.

Taber, D. F., Fessel, J. P., \& Roberts, L. J., 2nd. (2004). A nomenclature system for isofurans. Prostaglandins \& Other Lipid Mediators, 73, 47-50. https://doi.org/10.1016/ S0090-6980(97)00005-1.

Taş, N. G., \& Gökmen, V. (2017). Phenolic compounds in natural and roasted nuts and their skins: A brief review. Current Opinion in Food Science, 14, 103-109. https://doi. 
$\operatorname{org} / 10.1016 /$ j.cofs.2017.03.001.

Thomsen, M. K., Kristensen, D., \& Skibsted, L. H. (2000). Electron spin resonance spec troscopy for determination of the oxidative stability of food lipids. Journal of the American Oil Chemists Society, 77(7), 725-730.

Thurnhofer, S., \& Vetter, W. (2005). A gas chromatography/electron ionization-mass spectrometry-selected ion monitoring method for determining the fatty acid pattern in food after formation of fatty acid methyl esters. Journal of Agricultural and Food Chemistry, 53, 8896-8903. https://doi.org/10.1021/jf051468u.

Uquiche, E., Jeréz, M., \& Ortíz, J. (2008). Effect of pretreatment with microwaves on mechanical extraction yield and quality of vegetable oil from Chilean hazelnuts
(Gevuina avellana Mol). Innovative Food Science and Emerging Technologies, 9, 495-500. https://doi.org/10.1016/j.ifset.2008.05.004.

Velasco, J., Andersen, M. L., \& Skibsted, L. H. (2004). Evaluation of oxidative stability of vegetable oils by monitoring the tendency to radical formation. A comparison of electron spin resonance spectroscopy with the Rancimat method and differential scanning calorimetry. Food Chemistry, 85, 623-632. https://doi.org/10.1016/j. foodchem.2003.07.020.

Wangensteen, H., Samuelsen, A. B., \& Malterud, K. E. (2004). Antioxidant activity in extracts from coriander. Food Chemistry, 88, 293-297. https://doi.org/10.1016/j. foodchem.2004.01.047. 